\title{
A cohomological approach to immersed submanifolds via integrable systems
}

\author{
J. de Lucas ${ }^{1}$. A. M. Grundland ${ }^{2,3}$ \\ Published online: 24 August 2018 \\ (c) The Author(s) 2018
}

\begin{abstract}
A geometric approach to immersion formulas for soliton surfaces is provided through new cohomologies on spaces of special types of $\mathfrak{g}$-valued differential forms. We introduce Poincaré-type lemmas for these cohomologies, which appropriately describe the integrability conditions of Lax pairs associated with systems of PDEs. Our methods clarify the structure and properties of the deformations and soliton surfaces for the aforesaid Lax pairs. Our findings allow for the generalization of the theory of soliton surfaces in Lie algebras to general soliton submanifolds. Techniques from the theory of infinite-dimensional jet manifolds and diffieties enable us to justify certain common assumptions of the theory of soliton surfaces. Theoretical results are illustrated through $\mathbb{C} P^{N-1}$ sigma models.
\end{abstract}

Keywords Cohomology $\cdot \mathbb{C} P^{N-1}$ sigma model $\cdot$ Generalized symmetries $\cdot \mathfrak{g}$-valued differential forms $\cdot \mathfrak{g}$-valued de Rham cohomology $\cdot$ Integrable systems $\cdot$ Immersion formulas $\cdot$ Soliton surfaces

Mathematics Subject Classification Primary 35Q53; Secondary 35Q58 · 53A05

\section{Introduction}

Integrable models and their deformations under types of dynamics have produced considerable interest in mathematics, physics, and biology (see [5,9,13,14,25,43,50,

J. de Lucas

javier.de.lucas@fuw.edu.pl

1 Department of Mathematical Methods in Physics, University of Warsaw, ul. Pasteura 5, 02-093 Warsaw, Poland

2 Centre de Recherches Mathématiques, Université de Montréal, Succ. Centre-Ville, CP 6128, Montreal, QC H3C 3J7, Canada

3 Department of Mathematics and Computer Science, Université du Québec à Trois-Rivières, CP 500, Trois-Rivières, QC G9A 5H7, Canada 
$52,53,55,57]$ for details). The motivation for this topic came from applications like the growth of crystals [50], quantum field theory models [13,50,52], or the motion of boundaries between regions of different viscosities and densities $[8,57]$.

The progress in the description of surfaces obtained from nonlinear PDEs, e.g. soliton or constant curvature surfaces $[4,60]$, has resulted in many new techniques and theoretical approaches. Some of the most relevant developments have occurred in the study of soliton surfaces immersed in Lie algebras via techniques from the theory of completely integrable systems $[7,10,12,15,18-21,54,58,59]$.

The use of a linear spectral problem (LSP) to represent a moving frame on a soliton surface has yielded many findings concerning their intrinsic geometric properties [2, $4,35,40,48,49]$. The spectral parameter in the LSP describes deformations of soliton surfaces preserving their properties in such a way that integrable surfaces come in a family [4]. These surfaces are characterized by fundamental forms whose coefficients satisfy the Gauss-Weingarten and Gauss-Mainardi-Codazzi equations. It has recently proved fruitful to apply such a characterization of soliton surfaces to $\mathbb{C} P^{N-1}$ sigma models via their immersion formulas in Lie algebras. They have also been shown to play an essential role in many other problems of a physical nature (see $[2,23,24,29$, $46,65]$ and references therein).

The construction of the soliton surfaces related to the completely integrable $\mathbb{C} P^{N-1}$ sigma model has been accomplished by representing the Euler-Lagrange equations for this model as a conservation law which in turn provides a closed $\mathfrak{s u}(N)$-valued differential one-form on the surfaces. This is the so-called generalized Weierstrass formula for immersion $[33,39]$.

The construction of smooth orientable soliton surfaces related to completely integrable models in the sense of admitting a LSP problem was pioneered by Sym [58,59]. His technique exploits the conformal invariance of the zero-curvature representation of the LSP relative to the spectral parameter [4]. Another approach for determining such surfaces, formulated by Cieśliński and Doliwa [10,12,18], uses gauge symmetries of the LSP. Fokas and Gel'fand [20,21] developed a third approach by using the LSP for integrable systems and their Lie symmetries to derive families of soliton surfaces. In all cases, soliton surfaces are described through the so-called immersion formulas. Recently, a reformulation and extension of the Fokas-Gel'fand immersion formula has been performed through generalized vector fields and their actions on jet spaces [26-29]. This extension has provided the necessary and sufficient conditions for the existence of soliton surfaces in terms of the symmetries of the LSP and integrable models. It has also described the relations between the previously known immersion formulas.

Cohomological techniques have already been applied to the study of zero curvature representations and spectral parameters $[37,46]$. This paper aims to provide a unifying approach to immersion formulas via cohomological and geometric techniques. Our procedure provides simple geometric proofs for previous theoretical results on the topic, simplifies the theoretical expressions for immersion formulas via geometric structures, and extends the formalism of immersion formulas to create a theory of immersion formulas for soliton submanifolds and generalized Lax pairs with many potential applications (see e.g. $[1,3,11,34,44,64])$. 
Let $\mathfrak{g}$ be a Lie algebra and let $M, N$ be manifolds. Consider an integrable system of PDEs whose independent and dependent variables are coordinates on $M$ and $N$ respectively. The first objective of the paper is to give a more precise description of the standard objects appearing in the study of soliton surfaces for our system of PDEs, e.g. its LSPs, deformations, and immersion formulas, by the hereafter defined parametrized $\mathfrak{g}$-valued differential forms on-shell on $M$. In brief, a parametrized $\mathfrak{g}$-valued differential form on-shell is a family of $\mathfrak{g}$-valued differential forms on $M$ parametrized by particular solutions of the integrable system of PDEs and the spectral parameter $\lambda$ of a corresponding LSP. More generally, the parametrized $\mathfrak{g}$-valued differential forms on $M$ are $\mathfrak{g}$-valued differential forms on $M$ described by arbitrary functions from $M$ to $N$. The parametrized $\mathfrak{g}$-valued differential forms will also play a relevant role in the description of Lax pairs and immersion formulas. If not otherwise stated, it is assumed that the independent variables of systems of PDEs and all types of differential forms are defined on $M$.

The space of parametrized $\mathfrak{g}$-valued differential forms, say $\Omega_{\mathcal{N}}(M) \otimes \mathfrak{g}$, is endowed with a differential $\mathbf{d}$ leading to a cochain complex

$$
C_{\mathcal{N}^{(}}^{\infty}(M) \otimes \mathfrak{g} \stackrel{\mathbf{d}}{\longrightarrow} \Omega_{\mathcal{N}^{(}}^{1}(M) \otimes \mathfrak{g} \stackrel{\mathbf{d}}{\longrightarrow} \cdots \stackrel{\mathbf{d}}{\longrightarrow} \Omega_{\mathcal{N}^{m}}^{m}(M) \otimes \mathfrak{g} \stackrel{\mathbf{d}}{\longrightarrow} 0
$$

where $\Omega_{\mathcal{N}^{\prime}}^{k}(M) \otimes \mathfrak{g}$, with $k \in \mathbb{Z}$, is the space of parametrized $\mathfrak{g}$-valued differential $k$-forms (see [47] for details on cochain complexes). A LSP problem is then proved to amount to a parametrized $\mathfrak{g}$-valued differential one-form $\omega \in \Omega_{\mathcal{N}}^{1}(M) \otimes \mathfrak{g}$. This allows us to define a second operator $\mathbf{d}_{2 \omega}$ on the above cochain complex satisfying that $\mathbf{d}_{2 \omega} \omega$ vanishes exactly on the solutions of the system of PDEs under consideration. We then say that $\mathbf{d}_{2 \omega} \omega=0$ on-shell.

The space $\Omega_{\mathcal{S}}(M) \otimes \mathfrak{g}$ of parametrized $\mathfrak{g}$-valued differential forms on-shell can be endowed with two differentials $\mathbf{d}$ and $\mathbf{d}_{2 \omega}$ inducing new cochain complexes

$$
C_{\mathcal{S}}^{\infty}(M) \otimes \mathfrak{g} \stackrel{\mathbf{d}, \mathbf{d}_{2 \omega}}{\longrightarrow} \Omega_{\mathcal{S}}^{1}(M) \otimes \mathfrak{g} \stackrel{\mathbf{d}, \mathbf{d}_{2 \omega}}{\longrightarrow} \cdots \stackrel{\mathbf{d}, \mathbf{d}_{2 \omega}}{\longrightarrow} \Omega_{\mathcal{S}}^{m}(M) \otimes \mathfrak{g} \stackrel{\mathbf{d}_{2 \omega}}{\longrightarrow} 0
$$

where $\Omega_{\mathcal{S}}^{k}(M) \otimes \mathfrak{g}$, with $k \in \mathbb{Z}$, is the space of parametrized $\mathfrak{g}$-valued differential $k$-forms on-shell. In other words, $\mathbf{d}_{2 \omega}^{2}=0$ and $\mathbf{d}^{2}=0$ on $\Omega_{\mathcal{S}}(M) \otimes \mathfrak{g}$. We extend the Poincaré Lemma for the standard de Rham cohomology to this new realm so as to prove that $\vartheta \in \Omega_{\mathcal{S}}^{k}(M) \otimes \mathfrak{g}$ is $\mathbf{d}_{2 \omega}$-closed, i.e. $\mathbf{d}_{2 \omega} \vartheta=0$, if and only if it is locally exact, i.e. for every $p \in M$ there exists an open $U \subset M$ containing $p$ and $\varphi \in \Omega_{\mathcal{S}}^{k-1}(U) \otimes \mathfrak{g}$ such that $\vartheta=\mathbf{d}_{2 \omega} \varphi$ on $U$.

Next, we prove that a deformation of the LSP described by $\omega$ is equivalent to a $\mathbf{d}_{2 \omega}$-closed parametrized $\mathfrak{g}$-valued differential one-form on-shell $\Upsilon$, i.e. $\mathbf{d}_{2 \omega} \Upsilon=0$. The immersion formulas of Cieśliński-Doliwa and Fokas-Gel'fand provide particular solutions $\Upsilon$ of this equation for $M=\mathbb{R}^{2}$. Since $\mathbf{d}_{2 \omega}^{2}=0$, every $\mathbf{d}_{2 \omega} S$, with $S \in C_{\mathcal{S}}^{\infty}(M) \otimes \mathfrak{g}$, is a $\mathbf{d}_{2 \omega}$-closed parametrized $\mathfrak{g}$-valued differential one-form onshell. Hence, every $\Upsilon \in \Omega_{\mathcal{S}}^{1}(M) \otimes \mathfrak{g}$ such that $\Upsilon:=\mathbf{d}_{2 \omega} S$ gives rise to a deformation of the LSP related to $\omega$. A simple lemma allows us to retrieve the Cieśliński-Doliwa immersion formula from this latter fact. The Fokas-Gel'fand and Sym-Tafel immer- 
sion formulas can be explained in a slightly more involved but similar way. Many other results can be retrieved from simple geometric considerations.

Geometrically, we show that the immersion formulas induced by an infinitesimal deformation $\Upsilon$ take the form $F=\operatorname{Ad}_{\Phi^{-1}} \Upsilon$, where $\Phi$ stands for a family of solutions for the LSP induced by $\omega$ parametrized by its spectral parameter and solutions of the associated system of PDEs. Since the corresponding deformations are given by a $\mathbf{d}_{2 \omega^{-}}$ closed $\Upsilon$, our extended Poincaré Lemmas ensure that $\Upsilon$ is locally exact, i.e. $\Upsilon=\mathbf{d}_{2 \omega} S$ for an $S \in C_{\mathcal{S}}^{\infty}(M) \otimes \mathfrak{g}$. The function $S$ permits us to obtain the immersion formula associated with $\Upsilon$ for all known immersion formulas. For instance, our simple Lemma 6.1 enables us to retrieve the Cieśliński-Doliwa immersion formula immediately as $F=\operatorname{Ad}_{\Phi^{-1}} \mathbf{d}_{2 \omega} S=\mathbf{d A d} \Phi_{\Phi^{-1}} S$.

Different types of systems of PDEs appearing in the study of immersion formulas can be described as different types of parametrized $\mathfrak{g}$-valued differential forms. Our cohomologies describe the integrability conditions for such systems of PDEs. Local solutions are related to closed forms and global solutions to exact ones. Hence, cohomology groups related reflect the nature of the solutions of PDEs.

Results on the characterization of $\lambda$-conformal symmetries, symmetries of the system of PDEs, the existence of gauges for immersion formulas, and the presence of gauge transformations are easily proved by using our cohomologies.

The parametrized $\mathfrak{g}$-valued differential forms can be generated by a class of $\mathfrak{g}$-valued differential forms on jet spaces, the here called spectral differential forms [46]. Our cohomological procedures can be modified to deal with this jet approach by adapting known techniques from the theory of infinite-dimensional jet manifolds, diffieties and cohomology $[37,46,62,63]$. Particularly, this retrieves the jet approach to immersion formulas given in [27].

Finally, all previous results are coordinate free and they do not depend on the base manifold $M$ of the initial PDE under study. Hence, the whole theory can be extended to immersion formulas and Lax pairs on any $M$, e.g. to immersion formulas for submanifolds in Lie algebras [3,11].

This paper is organized as follows. Section 2 summarizes relevant results on jet bundles and the geometry of differential equations to be used hereafter. Sections 3 and 4 describe known and new cohomologies used in this work. The basic facts on LSPs and their description in terms of our new types of $\mathfrak{g}$-valued differential forms are introduced in Sect. 5. Subsequently, the theory of immersion formulas for general soliton submanifolds through cohomological and geometrical techniques is addressed in Sect. 6. The relation between different immersion formulas, e.g. the previously described multidimensional generalizations of the Sym-Tafel (ST), Cieśliński-Doliwa (CD) and Fokas-Gel'fand (FG) formulas, are detailed in Sect. 7. Next, Sect. 8 is concerned with the special case of immersion formulas related to Lax pairs describing solutions of PDEs obeying a certain boundary condition. Our techniques are illustrated through soliton surfaces for $\mathbb{C} P^{N-1}$ sigma models in Sect. 9. The main findings of the paper and topics for further research are summarized in Sect. 10. 


\section{On classical and generalized Lie symmetries}

This section reviews the main notions to be used hereafter: finite and infinitedimensional jet bundles, Lie symmetries, and related properties (see [51,62,63] for details). The summation convention over repeated indexes is hereafter employed.

We write $\overline{a, b}:=\{a, a+1, \ldots, b-1, b\}$ for $a<b \in \mathbb{Z}$. As previously, $M$ and $N$ are manifolds whose coordinates are the independent and dependent variables, respectively, of the system of PDEs under consideration. More specifically, $M$ and $N$ have local coordinates $x^{\alpha}$, for $\alpha \in \overline{1, m}$, and $u^{i}$, with $i \in \overline{1, n}$, correspondingly.

Let $J^{p}$ be the $p$-order jet bundle related to the trivial bundle $(N \times M, M, \pi$ : $N \times M \rightarrow M$ ) where $\pi$ is the projection onto $M$. As usual, we define $J^{0}:=M \times N$. The coordinates of $J^{p}$ are given by $x^{\alpha}, u^{i}$, and $u_{J}^{i}$, where $J:=\left(j_{1}, \ldots, j_{p}\right)$ is a multi-index with $1 \leq j_{1} \leq \cdots \leq j_{p} \leq m$ and length $|J|=p$. If $|J|=0$, then we set $u_{J}^{i}:=u^{i}$. We denote by $j_{x}^{p} u:=\left(x, u_{J}\right)$ a generic element of $J^{p}$. Given a section $s \in \Gamma(\pi)$, say $s(x):=(x, u(x))$, its prolongation to $J^{p}$ is denoted by $j^{p} s$ and reads $j^{p} s(x):=\left(x, u(x), \mathrm{d} u / \mathrm{d} x(x), \ldots, \mathrm{d}^{p} u(x) / \mathrm{d} x^{p}\right)$. Every function $u(x): M \rightarrow N$ amounts to a section $s_{u}: x \in M \mapsto(x, u(x)) \in M \times N$ of $J^{0}$ and vice versa. This motivates us to identify $u$ and its related section $s$ so as to simplify the notation. The Cartan distribution on $J^{p}$, let us say $\mathcal{C}^{p}$, is the smallest distribution on $J^{p}$ tangent to all $j^{p} s$ for an arbitrary $s \in \Gamma(\pi)[51]$.

The space $J^{\infty}$ is the inductive limit of all $J^{p}$ and the space of smooth functions on $J^{\infty}$ reads $C^{\infty}\left(J^{\infty}\right):=\cup_{p \in \overline{\mathbb{N}}} C^{\infty}\left(J^{p}\right)$ where $\overline{\mathbb{N}}:=\mathbb{N} \cup\{0\}$. Hence, the smooth functions on $J^{\infty}$ are smooth functions depending on a finite set of variables $x, u_{J}$. Previous construction endow $J^{\infty}$ with a manifold-like structure. Subsequently, $[u]$ stands for an arbitrary element of $J^{\infty}$. The Cartan distributions $\mathcal{C}^{p}$ can be extended to $J^{\infty}$ giving rise to an involutive distribution $\mathcal{C}$ on $J^{\infty}[41,62]$.

A vector field $X$ on $J^{0}$ can be written in local coordinates as

$$
X=\xi^{\alpha}(x, u) \partial_{\alpha}+\varphi^{i}(x, u) \partial_{i},
$$

where $\partial_{\alpha}:=\partial / \partial x^{\alpha}$, for $\alpha \in \overline{1, m}$, and $\partial_{i}:=\partial / \partial u^{i}$ for $i \in \overline{1, n}$. The vector field $X$ gives rise to its so-called prolonged vector field $\operatorname{pr} X$ on $J^{\infty}$ : the only vector field on $J^{\infty}$ leaving the space of vector fields taking values in $\mathcal{C}$ invariant (relative to the Lie bracket of vector fields) and projecting onto $X$. In particular, the total derivatives associated with the vector fields $\partial_{\alpha}$ read

$$
D_{\alpha}=\partial_{\alpha}+u_{J, \alpha}^{i} \frac{\partial}{\partial u_{J}^{i}}, \quad \alpha \in \overline{1, m},
$$

where $u_{J, \alpha}^{i}$ represents the variable $u_{J^{\prime}}^{i}$ with $J^{\prime}:=\left(j_{1}, \ldots, j_{p}, \alpha\right)$ and $J$ is an arbitrary multi-index. We also write $D_{J}:=D_{j_{1}} \ldots D_{j_{p}}$. Geometrically, $j^{p} X$ is the unique vector field on $J^{p}$ projecting onto $X$ on $J^{0}$ that leaves the vector fields taking values in $\mathcal{C}^{p}$ invariant (with respect to its action by Lie brackets). Alternatively, $j^{p} X$ can be defined as the restriction of $\operatorname{pr} X$ to functions in $C^{\infty}\left(J^{p}\right)$.

The vector fields on $J^{\infty}$ are not generally related to a one-parametric group of diffeomorphisms [63]. Nevertheless, there exist relevant types of vector fields on $J^{\infty}$ that admit a one-parametric group of transformations on $J^{\infty}$ (cf. [63]). 
Let us define on $J^{p}$ a system of PDEs in $m$ independent and $n$ dependent variables of the form

$$
\Delta^{\mu}\left(j_{x}^{p} u\right)=0, \quad \mu=\overline{1, s} .
$$

A particular solution of (2.3) is a map $u(x)$ from $M$ to $N$ whose prolongation to $J^{p}$, thought of as a section of $J^{0}$, satisfies (2.3).

The system of PDEs (2.3) determines a region $\mathcal{E} \subset J^{p}$ where the functions $\Delta^{\mu}$, with $\mu \in \overline{1, s}$, vanish. The system of PDEs (2.3) is hereupon assumed to be locally solvable, namely for each $j_{x}^{p} u \in \mathcal{E}$ there exists a solution $u(x)$ of the system (2.3) such that $j_{x}^{p} u$ belongs to the prolongation of $u(x)$ to $J^{p}$ [51, p. 158]. It is also assumed that the system (2.3) has maximal rank, i.e. the functions $\Delta^{\mu}$ are functionally independent and $\mathcal{E}$ is a submanifold of $J^{p}$ (cf. [51, p. 158]). These are assumptions satisfied by a large family of differential equations (cf. [51]).

A vector field $X$ on $J^{0}$ is a classical Lie point symmetry of the nondegenerate system of PDEs (2.3) if the prolongation of $X$ to $J^{p}$ is such that

$$
\left.j^{p} X \Delta^{\mu}\right|_{\mathcal{E}}=0, \quad \mu \in \overline{1, s} .
$$

The above formalism can be extended to $J^{\infty}$ by extending the system $(2.3)$ on $J^{p}$ to a system of equations on $J^{\infty}$ of the form

$$
\Delta^{\mu}([u]), \quad D_{J} \Delta^{\mu}([u])=0, \quad \mu \in \overline{1, s}, \quad \forall J .
$$

Recall that $\Delta^{\mu}$ only depends on the variables $u^{J}$ for $|J| \leq p$. Hence, the solutions of (2.5) are the extension to $J^{\infty}$ of particular solutions of $\Delta^{\mu}\left(j_{x}^{p} u\right)=0$. Equations (2.5) define a submanifold $\mathcal{E}^{\infty}$ of $J^{\infty}$ and the compositions of total derivatives, $D_{J}$, are tangent to it.

The Cartan distribution $\mathcal{C}$ on $J^{\infty}$ is the distribution generated by the vector fields $D_{\alpha}$ for $\alpha \in \overline{1, m}$. The Cartan distribution is involutive and the vector fields taking values in $\mathcal{C}$ are tangent to $\mathcal{E}^{\infty}$, which allows us to restrict $\mathcal{C}$ to $\mathcal{E}^{\infty}$ giving rise to the distribution $\left.\mathcal{C}\right|_{\mathcal{E}^{\infty}}$ on $\mathcal{E}^{\infty}$. The pair $\left(\mathcal{E}^{\infty},\left.\mathcal{C}\right|_{\mathcal{E}^{\infty}}\right)$ is called a diffiety [62].

To extend the number of symmetries, and thus of solutions, one looks for generalized symmetries. They exist only if the nonlinear equation (2.3) is integrable [49], i.e. it has been obtained as the compatibility of a Lax pair. To describe them, we make use of generalized vector fields. A generalized vector field is a vector field $X_{R}$ on $J^{\infty}$ ( [51, Definition 5.1]) of the form

$$
X_{R}:=R^{i}([u]) \frac{\partial}{\partial u^{i}},
$$

where the functions $R^{i} \in C^{\infty}\left(J^{\infty}\right)$ are arbitrary. The space of generalized vector fields is isomorphic to the space $\operatorname{sym}(\mathcal{C})$ of symmetries of the Cartan distribution $\mathcal{C}$ modulo Cartan vector fields, namely vector fields taking values in $\mathcal{C}$ [63]. Indeed, every vector field in $\operatorname{sym}(\mathcal{C})$ gives rise to a generalized vector field by restricting it to $C^{\infty}\left(J^{0}\right)$ and every generalized vector field $X_{R}$ can be extended to a unique vector field $\operatorname{pr} X$ on $J^{\infty}$ leaving invariant the space of vector fields taking values in $\mathcal{C}$, i.e. $\operatorname{pr} X$ induces an element of $\operatorname{sym}(\mathcal{C})$, whose action on $C^{\infty}\left(J^{0}\right)$ coincides with $X_{R}$. A 
vector field $X_{R}$ is a generalized symmetry of the system of PDEs (2.3) if and only if $\operatorname{pr} X_{R}$ is tangent to $\mathcal{E}^{\infty}[51]$.

\section{Cohomologies on parametrized $\mathfrak{g}$-valued differential forms}

This section generalises the de Rham cohomology of differential forms to cohomologies for differential forms taking values in Lie algebras parametrized by sections of a fiber bundle $(M \times N, M, \pi)$ and a parameter. We develop analogues for these new cohomologies of the Poincaré Lemma for de Rham cohomology. These results will later play a key role in studying and extending Lax pairs and immersion formulas for soliton surfaces within Lie algebras to immersion formulas. For completeness and clarity of our presentation, Theorem 3.1 is proved, although it can be regarded as a result of previous cohomological results on general diffieties (see $[46,63]$ ).

Let $\mathrm{d}$ stand for the standard exterior derivative on differential forms. The space $\Omega(M) \otimes \mathfrak{g}$ of $\mathfrak{g}$-valued differential forms on $M$ admits a natural exterior differential $\mathbf{d}: \Omega(M) \otimes \mathfrak{g} \rightarrow \Omega(M) \otimes \mathfrak{g}$ such that $\mathbf{d}(\theta \otimes v):=(\mathrm{d} \theta) \otimes v$ for every $v \in \mathfrak{g}$ and $\theta \in \Omega(M)$. Then, $\mathbf{d}^{2}=0$ and $\mathbf{d}$ induces a cochain complex:

$$
C^{\infty}(M) \otimes \mathfrak{g} \stackrel{\mathbf{d}}{\longrightarrow} \Omega^{1}(M) \otimes \mathfrak{g} \stackrel{\mathbf{d}}{\longrightarrow} \Omega^{2}(M) \otimes \mathfrak{g} \stackrel{\mathbf{d}}{\longrightarrow} \cdots \stackrel{\mathbf{d}}{\longrightarrow} \Omega^{m}(M) \otimes \mathfrak{g} \stackrel{\mathbf{d}}{\longrightarrow} 0,
$$

where $\Omega^{k}(M) \otimes \mathfrak{g}$, with $k \in \mathbb{Z}$, is the space of $\mathfrak{g}$-valued differential $k$-forms on $M$.

We say that $\theta \in \Omega(M) \otimes \mathfrak{g}$ is d-closed if $\mathbf{d} \theta=0$ and $\mathbf{d}$-exact when $\theta=\mathbf{d} \vartheta$ for a $\vartheta \in \Omega(M) \otimes \mathfrak{g}$. If $Z_{\mathrm{dR}}^{k}(M, \mathfrak{g})$ and $B_{\mathrm{dR}}^{k}(M, \mathfrak{g})$ are the spaces of $\mathbf{d}$-closed and d-exact $\mathfrak{g}$-valued differential $k$-forms, then $B_{\mathrm{dR}}^{k}(M, \mathfrak{g}) \subset Z_{\mathrm{dR}}^{k}(M, \mathfrak{g})$, and we define

$$
\mathrm{H}_{\mathrm{dR}}^{k}(M, \mathfrak{g}):=\frac{Z_{\mathrm{dR}}^{k}(M, \mathfrak{g})}{B_{\mathrm{dR}}^{k}(M, \mathfrak{g})}, \quad k \in \overline{\mathbb{N}} .
$$

The Poincaré Lemma for standard differential forms can be easily extended to $\mathbf{d}$-closed elements of $\Omega(M) \otimes \mathfrak{g}$. Additionally, $\mathrm{H}_{\mathrm{dR}}^{k}(M, \mathfrak{g}) \simeq \bigoplus_{j=1}^{\operatorname{dim} \mathfrak{g}} \mathrm{H}_{\mathrm{dR}}^{k}(M)$ for $k \in \overline{\mathbb{N}}$.

The space $\Omega(M) \otimes \mathfrak{g}$ can be endowed with a new cohomology via the bilinear bracket $[\cdot \wedge \cdot]$ of $\mathfrak{g}$-valued differential forms on $M$ satisfying (see [38] for details)

$$
\left[\vartheta_{1} \otimes v_{1} \wedge \vartheta_{2} \otimes v_{2}\right]:=\vartheta_{1} \wedge \vartheta_{2} \otimes\left[v_{1}, v_{2}\right], \quad \forall \vartheta_{1} \otimes v_{1}, \vartheta_{2} \otimes v_{2} \in \Omega(M) \otimes \mathfrak{g}
$$

This prompts us to define a new cochain complex on $\Omega(M) \otimes \mathfrak{g}$.

Theorem 3.1 Assume $\omega \in \Omega^{1}(M) \otimes \mathfrak{g}$ and define

$$
\mathbf{d}_{\omega} \vartheta:=\mathbf{d} \vartheta-\frac{1}{2}[\omega \wedge \vartheta], \quad \forall \vartheta \in \Omega(M) \otimes \mathfrak{g} .
$$

If $\mathbf{d}_{\omega} \omega=0$, then $\mathbf{d}_{2 \omega}^{2}=0$ and $\mathbf{d}_{2 \omega}$ induces a cochain complex

$$
C^{\infty}(M) \otimes \mathfrak{g} \stackrel{\mathbf{d}_{2 \omega}}{\longrightarrow} \Omega^{1}(M) \otimes \mathfrak{g} \stackrel{\mathbf{d}_{2 \omega}}{\longrightarrow} \Omega^{2}(M) \otimes \mathfrak{g} \stackrel{\mathbf{d}_{2 \omega}}{\longrightarrow} \ldots \stackrel{\mathbf{d}_{2 \omega}}{\longrightarrow} \Omega^{m}(M) \otimes \mathfrak{g} \stackrel{\mathbf{d}_{2 \omega}}{\longrightarrow} 0 .
$$


Proof Assume that $\vartheta \in \Omega^{k}(M) \otimes \mathfrak{g}$ with $k \in \overline{\mathbb{N}}$. Since $\omega \in \Omega^{1}(M) \otimes \mathfrak{g}$ in view of the definition of $\mathbf{d}_{2 \omega}$, and the Lie bracket on $\mathfrak{g}$-valued differential forms, it follows that $\mathbf{d} \vartheta-[\omega \wedge \vartheta]$ is a $\mathfrak{g}$-valued differential $(k+1)$-form. Hence, the sequence of linear morphisms (3.1) is well defined.

Let us prove that $\mathbf{d}_{2 \omega}^{2}=0$. By evaluating $\mathbf{d}_{2 \omega}^{2}$ on $\vartheta$, we obtain

$$
\begin{aligned}
\mathbf{d}_{2 \omega}^{2} \vartheta & =-[\mathbf{d} \omega \wedge \vartheta]+[\omega \wedge \mathbf{d} \vartheta]-[\omega \wedge(\mathbf{d} \vartheta-[\omega \wedge \vartheta])] \\
& =-[\mathbf{d} \omega \wedge \vartheta]+[\omega \wedge[\omega \wedge \vartheta]]
\end{aligned}
$$

Since $\mathbf{d} \omega=\frac{1}{2}[\omega \wedge \omega]$ by assumption, it follows that

$$
\mathbf{d}_{2 \omega}^{2} \vartheta=-\frac{1}{2}[[\omega \wedge \omega] \wedge \vartheta]+[\omega \wedge[\omega \wedge \vartheta]]
$$

Let us set $\mathrm{d} x^{J}:=\mathrm{d} x^{j_{1}} \wedge \cdots \wedge \mathrm{d} x^{j_{|J|}}$. In local coordinates $\omega=\mathrm{d} x^{\alpha} \otimes v_{\alpha}$ and $\vartheta=\mathrm{d} x^{J} \otimes v_{J}$ for certain vectors $v_{\alpha}, v_{J} \in \mathfrak{g}$. Hence,

$$
\begin{aligned}
\mathbf{d}_{2 \omega}^{2} \vartheta= & \mathrm{d} x^{\alpha} \wedge \mathrm{d} x^{\beta} \wedge \mathrm{d} x^{J} \otimes \frac{1}{2}\left(-\left[\left[v_{\alpha} \wedge v_{\beta}\right] \wedge v_{J}\right]\right. \\
& \left.+\left[v_{\alpha} \wedge\left[v_{\beta} \wedge v_{J}\right]\right]+\left[v_{\alpha} \wedge\left[v_{\beta} \wedge v_{J}\right]\right]\right) .
\end{aligned}
$$

The Jacobi identity for the Lie bracket in $\mathfrak{g}$ implies that

$$
\mathbf{d}_{2 \omega}^{2} \vartheta=\mathrm{d} x^{\alpha} \wedge \mathrm{d} x^{\beta} \wedge \mathrm{d} x^{J} \otimes \frac{1}{2}\left(\left[v_{\beta},\left[v_{\alpha}, \wedge v_{J}\right]+\left[v_{\alpha},\left[v_{\beta}, v_{J}\right]\right]\right) .\right.
$$

Since the coefficients relative to the indices $\alpha, \beta, J$ of the above $\mathfrak{g}$-valued differential $(k+1)$-form are symmetric relative to the interchange of $\alpha$ and $\beta$, it follows that $\mathbf{d}_{2 \omega}^{2} \vartheta=0$. As this result remains true for any $\vartheta$ and any coordinated open subset of $M$, it turns out that $\mathbf{d}_{2 \omega}^{2}=0$.

Now, $\theta \in \Omega(M) \otimes \mathfrak{g}$ is said to be $\mathbf{d}_{2 \omega}$-closed if $\mathbf{d}_{2 \omega} \theta=0$ and $\mathbf{d}_{2 \omega}$-exact when $\theta=\mathbf{d}_{2 \omega} \vartheta$ for a certain $\vartheta \in \Omega(M) \otimes \mathfrak{g}$. Let $Z_{2 \omega}^{k}(M, \mathfrak{g})$ and $B_{2 \omega}^{k}(M, \mathfrak{g})$ be the spaces of $\mathbf{d}_{2 \omega}$-closed and $\mathbf{d}_{2 \omega}$-exact $\mathfrak{g}$-valued differential $k$-forms, respectively. Since $\mathbf{d}_{2 \omega}^{2}=0$, it follows that $B_{2 \omega}^{k}(M, \mathfrak{g}) \subset Z_{2 \omega}^{k}(M, \mathfrak{g})$ and we can define

$$
\mathrm{H}_{2 \omega}^{k}(M, \mathfrak{g}):=\frac{Z_{2 \omega}^{k}(M, \mathfrak{g})}{B_{2 \omega}^{k}(M, \mathfrak{g})}, \quad k \in \overline{\mathbb{N}} .
$$

The Poincaré Lemma can be again extended to $\mathfrak{g}$-valued differential forms on $M$ relative to $\mathbf{d}_{\omega}$ and $\mathbf{d}_{2 \omega}$. The first generalization is immediate and, in particular, $\mathrm{H}_{2 \omega}^{k}(M, \mathfrak{g}) \simeq \oplus_{j=1}^{\operatorname{dim} \mathfrak{g}} \mathrm{H}_{\mathrm{dR}}^{k}(M)$ for $k \in \overline{\mathbb{N}}$. Meanwhile, a partial generalization of the Poincaré Lemma for the cohomology $\mathbf{d}_{2 \omega}$ is given by the following theorem.

Theorem 3.2 Every $\mathbf{d}_{2 \omega}$-closed differential one-form $\vartheta \in \Omega^{1}(M) \otimes \mathfrak{g}$ is locally $\mathbf{d}_{2 \omega^{-}}$ exact, namely for every $p \in M$ there exists and open $U \ni p$ and $\theta \in C^{\infty}(U) \otimes \mathfrak{g}$ such that $\mathbf{d}_{2 \omega} \theta=\vartheta$ on $U$. 
Proof The condition $\mathbf{d}_{2 \omega} \theta=\vartheta$, with $\theta \in C^{\infty}(M) \otimes \mathfrak{g}$, amounts to

$$
\mathbf{d} \theta=[\omega \wedge \theta]+\vartheta
$$

By using the zero-curvature condition (ZCC) for this system of first-order PDEs in the unknown $\theta$, it follows that the system has a local solution if and only if $\mathbf{d}([\omega \wedge \theta]+\vartheta)=$ 0 . Since $\mathbf{d}_{\omega} \omega=0$, it follows that

$$
\begin{gathered}
\mathbf{d}([\omega \wedge \theta]+\vartheta)=[\mathbf{d} \omega \wedge \theta]-[\omega \wedge \mathbf{d} \theta]+\mathbf{d} \vartheta=\frac{1}{2}[[\omega \wedge \omega] \wedge \theta] \\
-[\omega \wedge([\omega \wedge \theta]+\vartheta)]+\mathbf{d} \vartheta=\mathbf{d}_{2 \omega} \vartheta .
\end{gathered}
$$

Hence, $\mathbf{d}_{2 \omega} \theta=\vartheta$ for a certain locally defined $\theta$ if and only if $\mathbf{d}_{2 \omega} \vartheta=0$.

The d-closedness of elements of $\Omega(M) \otimes \mathfrak{g}$ is related to the existence of solutions to certain systems of PDEs. For instance, if $\mathbf{d}_{\omega} \omega=0$, then

$$
\begin{aligned}
& \forall p \in M, \exists U \ni p, \exists F \in C^{\infty}(U) \otimes \mathfrak{g}, \quad \mathbf{d} F=\Upsilon \Longleftrightarrow \Upsilon \in Z_{\mathrm{dR}}^{1}(M, \mathfrak{g}), \\
& \forall p \in M, \exists U \ni p, \exists g \in C^{\infty}(U) \otimes G, \quad \mathbf{d} g=R_{g} \omega, \quad \omega \in C^{\infty}(U) \otimes \mathfrak{g} \\
& \quad \Longleftrightarrow \omega \in Z_{2 \omega}^{1}(M, \mathfrak{g}), \\
& \forall p \in M, \exists U \ni p, \exists F \in C^{\infty}(U) \otimes \mathfrak{g}, \quad \mathbf{d} F-[\omega, F]=\Upsilon \Longleftrightarrow \Upsilon \in Z_{2 \omega}^{1}(M, \mathfrak{g}) .
\end{aligned}
$$

The first and third cases are immediate. The second one follows from the fact that $G$ and $\mathfrak{g}$ are matrix Lie groups and Lie algebras, respectively, and $\mathbf{d}$ acts on them as a de Rham differential on their matrix entries.

The above results can be generalized to the hereafter called parametrized $\mathfrak{g}$-valued differential forms. These differential forms will appear naturally in the study of Lax pairs and immersion formulas. We define $\mathcal{N}:=J^{p} \times \Lambda$, where $\Lambda$ is a one-dimensional submanifold of $\mathbb{C}$ coordinated by the variable $\lambda$.

Definition 3.3 A parametrized $\mathfrak{g}$-valued differential form is a family of $\mathfrak{g}$-valued differential forms on $M$, say

$$
\chi(u(x), \lambda)=\chi_{J}(u(x), \lambda) \mathrm{d} x^{J}, \quad \chi_{J}(u(x), \lambda) \in C^{\infty}(M),
$$

parametrized by arbitrary sections $u \in \Gamma(\pi)$ and $\lambda \in \Lambda$. A parametrized $\mathfrak{g}$-valued differential form on-shell is a family of $\mathfrak{g}$-valued differential forms (3.4) where $u \in$ $\Gamma(\pi)$ is a solution of $\Delta\left(j_{x}^{p} u\right)=0$. We write $\Omega_{\mathcal{N}}(M) \otimes \mathfrak{g}\left(\operatorname{resp} . \Omega_{\mathcal{S}}(M) \otimes \mathfrak{g}\right)$ for the space of parametrized $\mathfrak{g}$-valued differential forms (resp. on-shell).

The space $\Omega_{\mathcal{N}}(M) \otimes \mathfrak{g}$ admits a grading $\Omega_{\mathcal{N}}(M) \otimes \mathfrak{g}=\bigoplus_{k \in \mathbb{Z}} \Omega_{\mathcal{N}}^{k}(M) \otimes \mathfrak{g}$ compatible with the exterior product of parametrized $\mathfrak{g}$-valued differential forms that is defined in the natural way. A similar grading can be applied to parametrized $\mathfrak{g}$-valued differential equations on-shell. The elements of each $\Omega_{\mathcal{N}}^{k}(M) \otimes \mathfrak{g}\left(\operatorname{resp} . \Omega_{\mathcal{S}}^{k}(M) \otimes \mathfrak{g}\right)$ are called parametrized $\mathfrak{g}$-valued differential k-forms (resp. on-shell).

The following theorem allows us to define two cohomologies on $\Omega_{\mathcal{S}}(M) \otimes \mathfrak{g}$. It is indeed a natural generalization of Theorem 3.1 to $\Omega_{\mathcal{N}}(M) \otimes \mathfrak{g}$ and $\Omega_{\mathcal{S}}(M) \otimes \mathfrak{g}$. 
Theorem 3.4 Let $\omega \in \Omega_{\mathcal{N}}^{1}(M) \otimes \mathfrak{g}$ be such that $\mathbf{d}_{\omega} \omega=0$ only on particular solutions of (2.3). Let $\mathbf{d}$ and $\mathbf{d}_{2 \omega}$ be the linear operators on $\Omega_{\mathcal{N}}(M) \otimes \mathfrak{g}$ acting on elements $\vartheta=\vartheta_{J} \mathrm{~d} x^{J}$ in the form

$$
\mathbf{d} \vartheta:=\left(d_{\alpha} \vartheta_{J}\right) \mathrm{d} x^{\alpha} \wedge \mathrm{d} x^{J}, \quad \mathbf{d}_{2 \omega} \vartheta:=\mathbf{d} \vartheta-[\omega \wedge \vartheta]
$$

where $\left(d_{\alpha} \vartheta_{J}\right)(u(x), \lambda):=\partial_{\alpha}\left[\vartheta_{J}(u(x), \lambda)\right]$ and $\omega \in \Omega_{\mathcal{N}^{(}}^{1}(M) \otimes \mathfrak{g}$. The space $\Omega_{\mathcal{N}}(M) \otimes \mathfrak{g}$ admits a cohomology induced by $\mathbf{d}$. Meanwhile, $\Omega_{\mathcal{S}}(M) \otimes \mathfrak{g}$ admits two cohomologies given by $\mathbf{d}$ and $\mathbf{d}_{2 \omega}$.

Proof In view of (3.5), the differential $\mathbf{d}$ of a parametrized $\mathfrak{g}$-valued differential $k$-form is a parametrized $\mathfrak{g}$-valued differential $(k+1)$-form. Additionally, the commutativity of partial derivatives $\partial_{\alpha}, \partial_{\beta}$, for $\alpha, \beta \in \overline{1, m}$, and its action on the differential functions $\vartheta_{J}(u(x), \lambda)$ for each fixed $u(x)$ implies that $\left[d_{\alpha}, d_{\beta}\right]=0$. Hence,

$$
\mathbf{d}^{2} \vartheta=\left(d_{\beta} d_{\alpha} \vartheta_{J}\right)(u(x), \lambda) \mathrm{d} x^{\beta} \wedge \mathrm{d} x^{\alpha} \wedge d x^{J}=0
$$

because the coefficients of $\mathbf{d}^{2} \vartheta$, i.e. $d_{\beta} d_{\alpha} \vartheta_{J}$, are symmetric relative to the interchange of $\alpha$ and $\beta$. Additionally,

$$
\begin{aligned}
\mathbf{d}\left[\omega_{1} \wedge \omega_{2}\right]= & {\left[\mathbf{d} \omega_{1} \wedge \omega_{2}\right]+(-1)^{k}\left[\omega_{1} \wedge \mathbf{d} \omega_{2}\right] } \\
& \forall \omega_{1} \in \Omega_{\mathcal{N}^{S}}(M) \otimes \mathfrak{g}, \quad \forall \omega_{2} \in \Omega_{\mathcal{N}}(M) \otimes \mathfrak{g} .
\end{aligned}
$$

Following the same ideas of Theorem 3.1 and using the previous fact, we see that $\mathbf{d}_{2 \omega}$ induces a cohomology on $\Omega_{\mathcal{S}}(M) \otimes \mathfrak{g}$.

The above theorem denotes different types of differentials in the same way. This simplifies the notation and it is not misleading since the meaning of $\mathbf{d}$ and $\mathbf{d}_{2 \omega}$ is clear taking into account on which kind of $\mathfrak{g}$-valued differential form the operator is acting on. The same idea will be applied to other new differentials described in Sect. 4. Similarly to previous cohomologies, one can define closeness and exactness relative to the operators $\mathbf{d}$ and $\mathbf{d}_{2 \omega}$. The spaces $Z_{\mathcal{S}}^{k}(M, \mathfrak{g}), B_{\mathcal{S}}^{k}(M, \mathfrak{g}), \mathrm{H}_{\mathcal{S}}^{k}(M, \mathfrak{g}), Z_{\mathcal{S}, 2 \omega}^{k}(M, \mathfrak{g})$, $B_{\mathcal{S}, 2 \omega}^{k}(M, \mathfrak{g}), \mathrm{H}_{\mathcal{S}, 2 \omega}^{k}(M, \mathfrak{g}) Z_{\mathcal{N}^{\mathcal{N}}}^{k}(M, \mathfrak{g}), B_{\mathcal{N}}^{k}(M, \mathfrak{g})$, and $\mathrm{H}_{\mathcal{N}}^{k}(M, \mathfrak{g})$ can be defined as for previous cohomologies. By applying Theorem 3.2 for each fixed $u(x)$ and $\lambda$, we get the following corollaries.

Corollary 3.5 Every $\mathbf{d}_{2 \omega}$-closed element of $\Omega_{\mathcal{S}}^{1}(M) \otimes \mathfrak{g}$ is locally $\mathbf{d}_{2 \omega}$-exact. Every d-closed element of $\Omega_{\mathcal{N}^{k}}^{k}(M) \otimes \mathfrak{g}$ or $\Omega_{\mathcal{S}}^{k}(M) \otimes \mathfrak{g}$ is locally d-exact.

\section{Spectral differential forms}

Parametrized $\mathfrak{g}$-valued differential forms of the previous section can be generated, in relevant cases, through a type of $\mathfrak{g}$-valued differential forms on spaces of infinite jets: the hereafter called spectral differential forms. Although the theoretical description of these new structures is more complicated than the description of parametrized $\mathfrak{g}$-valued 
differential forms, they offer numerous practical advantages in calculations, which justifies their introduction. In particular, this formalism permits us to use the powerful machinery of the theory of jet bundles to obtain Lie symmetries, immersion formulas, and other related structures. Relevantly, this formalism will fill some theoretical details in the jet formalism of the immersion formulas given in [26]. Spectral differential forms can be considered as a reformulation of the differential forms on diffieties in $[37,46]$. Moreover, Theorem 4.2 can be regarded as an analogue of results in the theory of bicomplexes for $\mathfrak{g}$-valued differential forms on diffieties (see $[37,46,63]$ ).

Definition 4.1 A spectral differential form is a family of $\mathfrak{g}$-valued differential forms on $J^{\infty}$ parametrized by a spectral parameter $\lambda \in \Lambda \subset \mathbb{C}$ for a certain submanifold $\Lambda \subset \mathbb{C}$ and taking the form

$$
\omega_{\lambda}:=\omega_{J}([u], \lambda) \mathrm{d} x^{J}
$$

where $\omega_{J}([u], \lambda)$ is any function in $C_{\lambda}^{\infty}\left(J^{\infty}\right):=\cup_{p \in \overline{\mathbb{N}}} C^{\infty}\left(J^{p} \times \Lambda\right)$. A spectral differential form on-shell is the restriction of a spectral differential form (4.1) to a $\lambda$-parametrized family of $\mathfrak{g}$-valued differential forms on $\mathcal{E}^{\infty}$. We write $\Omega_{\lambda}\left(J^{\infty}\right) \otimes \mathfrak{g}$ (resp. $\Omega_{\lambda}\left(\mathcal{E}^{\infty}\right) \otimes \mathfrak{g}$ ) for the space of spectral differential forms (resp. on-shell).

The space $\Omega_{\lambda}\left(J^{\infty}\right) \otimes \mathfrak{g}$ admits a grading $\Omega_{\lambda}\left(J^{\infty}\right) \otimes \mathfrak{g}=\bigoplus_{k \in \mathbb{Z}} \Omega_{\lambda}^{k}\left(J^{\infty}\right) \otimes \mathfrak{g}$ compatible with the exterior product of spectral differential forms defined in the natural way. A similar grading can be applied to spectral differential forms on-shell. The elements of each $\Omega_{\lambda}^{k}\left(J^{\infty}\right) \otimes \mathfrak{g}$ (resp. $\left.\Omega_{\lambda}^{k}\left(\mathcal{E}^{\infty}\right) \otimes \mathfrak{g}\right)$ are called spectral differential $k$-forms (resp. on-shell).

Every spectral differential form induces a unique parametrized $\mathfrak{g}$-valued differential form. Indeed, given (4.1) and a section $s \in \Gamma(\pi)$ of the form $s(x)=(x, u(x))$, a parametrized $\mathfrak{g}$-valued differential form is defined by $\omega(u(x), \lambda):=j^{\infty} s^{*} \omega_{\lambda}$. If $u(x)$ is a solution of our system of PDEs, then $\omega(u(x), \lambda)$ becomes a parametrized $\mathfrak{g}$-valued differential form on-shell. Meanwhile, a parametrized $\mathfrak{g}$-valued differential form need not come from a spectral differential form.

Since spectral differential forms give rise to parametrized $\mathfrak{g}$-valued differential forms, it becomes relevant how to apply the formalism for parametrized $\mathfrak{g}$-valued differential forms directly to spectral differential forms. This is accomplished by the following theorem, which provides an extension of the horizontal differential on the infinite-dimensional jet bundle $J^{\infty}$ (see [41]). This can be regarded as a generalization of cohomologies given in $[46,63]$ to spectral differential forms.

Theorem 4.2 Let $\omega \in \Omega_{\lambda}^{1}\left(J^{\infty}\right) \otimes \mathfrak{g}$ be such that $\mathbf{d}_{\omega} \omega=0$ on $\mathcal{E}^{\infty}$. Let $\mathbf{d}$ and $\mathbf{d}_{2 \omega}$ be the linear operators on $\Omega_{\lambda}\left(J^{\infty}\right) \otimes \mathfrak{g}$ acting on homogeneous elements $\vartheta=\vartheta_{J} \mathrm{~d} x^{J} \in$ $\Omega_{\lambda}^{k}\left(J^{\infty}\right) \otimes \mathfrak{g}$ given by

$$
\mathbf{d} \vartheta:=\left(D_{\alpha} \vartheta_{J}\right) \mathrm{d} x^{\alpha} \wedge \mathrm{d} x^{J}, \quad \mathbf{d}_{2 \omega} \vartheta:=\mathbf{d} \vartheta-[\omega \wedge \vartheta]
$$

The space $\Omega_{\lambda}\left(J^{\infty}\right) \otimes \mathfrak{g}$ admits a cohomology given by $\mathbf{d}$. The space $\Omega_{\lambda}\left(\mathcal{E}^{\infty}\right) \otimes \mathfrak{g}$ admits two cohomologies given by $\mathbf{d}$ and $\mathbf{d}_{2 \omega}$. 
Proof The formula (4.2) ensures that the differential $\mathbf{d}$ of a spectral differential $k$-form on $J^{\infty}$ is a spectral differential $(k+1)$-form. Since $\left[D_{\alpha}, D_{\beta}\right]=0$ on $J^{\infty}$ (cf. [51]),

$$
\mathbf{d}^{2} \vartheta=\left(D_{\beta} D_{\alpha} \vartheta_{J}\right) \mathrm{d} x^{\beta} \wedge \mathrm{d} x^{\alpha} \wedge d x^{J}=0
$$

because the coefficients of this spectral differential $(k+2)$-form, namely $D_{\beta} D_{\alpha} \vartheta_{J}$, are symmetric relative to the interchange of $\alpha$ and $\beta$. Hence, $\mathbf{d}$ gives rise to a cohomology in $\Omega_{\lambda}\left(J^{\infty}\right) \otimes \mathfrak{g}$. Meanwhile,

$$
\begin{aligned}
\mathbf{d}\left[\omega_{1} \wedge \omega_{2}\right]= & {\left[\mathbf{d} \omega_{1} \wedge \omega_{2}\right]+(-1)^{s}\left[\omega_{1} \wedge \mathbf{d} \omega_{2}\right], \quad \forall \omega_{1} \in \Omega_{\lambda}^{s}\left(J^{\infty}\right) \otimes \mathfrak{g} } \\
& \forall \omega_{2} \in \Omega_{\lambda}\left(J^{\infty}\right) \otimes \mathfrak{g} .
\end{aligned}
$$

The vector fields $D_{\alpha}$ are tangent to $\mathcal{E}^{\infty}$, which allows us to define the action of $D_{\alpha}$ on $C_{\lambda}^{\infty}\left(\mathcal{E}^{\infty}\right)$ and to restrict $\mathbf{d}$ and $\mathbf{d}_{2 \omega}$ to spectral differential forms on-shell. Moreover, since $\left[D_{\alpha}, D_{\beta}\right]=0$ on $\mathcal{E}^{\infty}$, it follows that $\mathbf{d}^{2}=0$, which gives rise to a differential and a cochain simplex on $\Omega_{\lambda}\left(\mathcal{E}^{\infty}\right) \otimes \mathfrak{g}$. This along with the definition (4.2) allows us to apply the ideas of the proof in Theorem 3.1 so as to prove that $\mathbf{d}_{2 \omega}$ gives rise to a cohomology in $\Omega_{\lambda}\left(\mathcal{E}^{\infty}\right) \otimes \mathfrak{g}$.

Let $H_{\mathrm{dR}}^{k}\left(J^{\infty}, \mathfrak{g}\right)$ and $H_{\mathrm{dR}}^{k}\left(\mathcal{E}^{\infty}, \mathfrak{g}\right)$ be the cohomologic groups induced by $\mathbf{d}$ on $\Omega_{\lambda}\left(J^{\infty}\right) \otimes \mathfrak{g}$ and $\Omega_{\lambda}\left(\mathcal{E}^{\infty}\right) \otimes \mathfrak{g}$. The proof of Theorem 4.2 and the form of $\mathbf{d}$ show that $H_{\mathrm{dR}}^{k}\left(J^{\infty}, \mathfrak{g}\right) \simeq \bigoplus_{j=1}^{\operatorname{dim} \mathfrak{g}} H_{\mathrm{dR}}^{k}\left(J^{\infty}\right)$. Since $M \times N$ is a strong deformation retract of $J^{\infty}$, then $H_{\mathrm{dR}}^{k}\left(J^{\infty}\right) \simeq H_{\mathrm{dR}}^{k}(M \times N)$ (see [22] for details).

It is worth commenting on the space on which the spectral differential form $\omega$ vanishes. The LSP for a system of PDEs gives rise to a parametrized $\mathfrak{g}$-valued differential form $\omega$ such that $\mathbf{d}_{\omega} \omega=0$ for every $\lambda \in \Lambda$ only on particular solutions of our system of PDEs. In practical applications, $\omega$ is then described by a spectral differential oneform, $\omega_{\lambda}$, vanishing for every $\lambda \in \Lambda$ on those points of $J^{\infty}$ projecting onto $\mathcal{E}$. Then, $\mathcal{E}^{\infty}$ is contained in this space and $\mathbf{d}_{\omega_{\lambda}} \omega_{\lambda}=0$ on $\mathcal{E}^{\infty}$.

Let us prove the following new Poincaré Lemma-type result, which is an extension of the Poincaré Lemma for the cohomology of horizontal forms on diffieties and infinite-dimensional jet bundles $J^{\infty}[63]$.

Theorem 4.3 Let $J^{0}$ and $\mathcal{E}^{\infty}$ be homotopic to $\mathbb{R}^{m}$. Then every $\mathbf{d}$-closed spectral differential form or spectral differential form on-shell is exact. Every $\mathbf{d}_{2 \omega}$-closed spectral differential one-form on-shell is locally exact.

Proof The differential operators $\mathbf{d}$ and $\mathbf{d}_{2 \omega}$ induce cochain complexes with respect to the gradings $\Omega_{\lambda}\left(J^{\infty}\right) \otimes \mathfrak{g} \simeq \oplus_{k \in \mathbb{Z}} \Omega_{\lambda}^{k}\left(J^{\infty}\right) \otimes \mathfrak{g}$ and $\Omega_{\lambda}\left(\mathcal{E}^{\infty}\right) \otimes \mathfrak{g} \simeq \oplus_{k \in \mathbb{Z}} \Omega_{\lambda}^{k}\left(\mathcal{E}^{\infty}\right) \otimes \mathfrak{g}$, i.e. we have the cochain complexes

$$
\begin{aligned}
& C_{\lambda}^{\infty}\left(J^{\infty}\right) \otimes \mathfrak{g} \stackrel{\mathbf{d}}{\longrightarrow} \Omega_{\lambda}^{1}\left(J^{\infty}\right) \otimes \mathfrak{g} \stackrel{\mathbf{d}}{\longrightarrow} \Omega_{\lambda}^{2}\left(J^{\infty}\right) \otimes \mathfrak{g} \stackrel{\mathbf{d}}{\longrightarrow} \cdots \\
& C_{\lambda}^{\infty}\left(\mathcal{E}^{\infty}\right) \otimes \mathfrak{g} \stackrel{\mathbf{d}, \mathbf{d}_{2} \omega}{\longrightarrow} \Omega_{\lambda}^{1}\left(\mathcal{E}^{\infty}\right) \otimes \mathfrak{g} \stackrel{\mathbf{d}, \mathbf{d}_{2} \omega}{\longrightarrow} \Omega_{\lambda}^{2}\left(\mathcal{E}^{\infty}\right) \otimes \mathfrak{g} \stackrel{\mathbf{d}, \mathbf{d}_{2} \omega}{\longrightarrow} \cdots
\end{aligned}
$$


For every fixed $\lambda$, the space $\Omega_{\lambda}^{k}\left(J^{\infty}\right) \otimes \mathfrak{g}$ is isomorphic to $\operatorname{dim} \mathfrak{g}$-copies of the so-called horizontal space

$$
\bar{\Lambda}^{k}\left(J^{\infty}\right):=\left\{\vartheta:=\vartheta_{J}([u]) \mathrm{d} x^{J}:[u] \in J^{\infty}, \vartheta_{J}([u]) \in \mathfrak{g}\right\}
$$

of horizontal differential forms on $J^{\infty}$. Moreover, these spaces give rise to a cochain complex for the so-called horizontal differential $d_{h}$ of the variational complex (see [51, Section 5.4]), which coincides with $\mathbf{d}$ when $\operatorname{dim} \mathfrak{g}=1$. Additionally $\mathbf{d}=\mathrm{d}_{h} \oplus \ldots \oplus \mathrm{d}_{h}$ (dim $\mathfrak{g}$ times). If a horizontal form is closed relative to $d_{h}$, then it is locally exact (cf. [51, Theorem 5.82]). Hence, a d-closed spectral differential form will be always locally d-exact.

The previous result can be extended to spectral differential forms on-shell by using the diffiety $\left(\mathcal{E}^{\infty},\left.\mathcal{C}\right|_{\mathcal{E}^{\infty}}\right)$. It is useful to note that, for a trivial differential equation $\mathcal{E}=J^{p}$, one has that $\left(\mathcal{E}^{\infty},\left.\mathcal{C}\right|_{\mathcal{E}}\right)=\left(J^{\infty}, \mathcal{C}\right)$ and the following commentaries retrieve the results of the previous paragraph. The space of spectral differential forms on-shell for a particular $\lambda$ is isomorphic to dim $\mathfrak{g}$-copies of the space of horizontal forms of the diffiety, $\Lambda_{h}\left(\mathcal{E}^{\infty}\right)$, and the restriction of the differential $\mathbf{d}$ to $\Omega_{\lambda}\left(\mathcal{E}^{\infty}\right) \otimes \mathfrak{g}$ is indeed the horizontal differential on the diffiety $\mathcal{E}^{\infty}$ (see [62] for details on this and following commentaries). As every closed horizontal differential form is locally closed relative to the differential of the diffiety, every $\mathbf{d}$-closed form on $\mathcal{E}^{\infty}$ is locally exact. Since $\mathcal{E}^{\infty}$ is homotopic to $\mathbb{R}^{m}$, every $\mathbf{d}$-closed spectral differential form on-shell is exact.

The proof for the local exactness of $\mathbf{d}_{2 \omega}$-closed spectral differential one-forms follows from writing the partial differential equations $\Upsilon=\mathbf{d}_{2 \omega} F$, for $\Upsilon \in \Omega_{\lambda}^{1}\left(\mathcal{E}^{\infty}\right) \otimes \mathfrak{g}$ in terms of $\mathbf{d}$. The obtained differential equation in $F$ is integrable if the system of PDEs on $\mathcal{E}^{\infty}$ is analytic and formally integrable, which amounts to $\mathbf{d}_{2 \omega} \Upsilon=0$ (cf. [42]). Hence, a local solution $F$ can be obtained and $\Upsilon$ becomes locally exact.

\section{LSPs and $\mathfrak{g}$-valued differential forms}

To analyse immersion formulas for soliton surfaces in Lie algebras and to generalize them to immersed soliton submanifolds in Lie algebras, we will describe integrability conditions in terms of cohomological techniques.

Let us consider an integrable (in the sense of having a LSP) system of PDEs on $J^{p}$ of the form

$$
\Delta\left(j_{x}^{p} u\right)=0
$$

for a function $\Delta: J^{p} \rightarrow \mathbb{R}^{s}$. Recall that the above system is assumed to have maximal rank and to be locally solvable.

Let $G$ be a matrix Lie group and let $\mathfrak{g}$ be its matrix Lie algebra. Consider that the LSP problem related to $(5.1)$ is given by a $(\lambda, u(x))$-parametrized family of systems of linear PDEs

$$
\partial_{\alpha} \Phi=U_{\alpha}(u(x), \lambda) \Phi, \quad \alpha \in \overline{1, m}, \quad \Phi \in G, \quad U_{\alpha}(u(x), \lambda) \in \mathfrak{g}, \quad \lambda \in \Lambda \subset \mathbb{C},
$$


whose integrability condition, the Zero-Curvature Condition (ZCC), i.e.

$$
\partial_{\beta} U_{\alpha}-\partial_{\alpha} U_{\beta}+\left[U_{\alpha}, U_{\beta}\right]=0, \quad \forall \lambda \in \Lambda, \quad \forall \alpha, \beta \in \overline{1, m}
$$

is satisfied if and only if $u(x)$ is a solution to (5.1). Let us describe this result cohomologically. In this respect, we define a parametrized $\mathfrak{g}$-valued differential one-form $\omega(u(x), \lambda):=U_{\alpha}(u(x), \lambda) \mathrm{d} x^{\alpha}$. Then, the Zero-Curvature Condition (ZCC) for (5.1) amounts to looking for the curves $u(x): M \rightarrow N$ ensuring that

$$
\mathbf{d}_{\omega} \omega:=\mathbf{d} \omega-\frac{1}{2}[\omega \wedge \omega]=0, \quad \forall \lambda \in \Lambda
$$

This equivalence appears as a consequence of writing (5.4) in coordinates, namely

$$
\begin{aligned}
0 & =\partial_{\beta} U_{\alpha} \mathrm{d} x^{\beta} \wedge \mathrm{d} x^{\alpha}-\frac{\left[U_{\alpha} \mathrm{d} x^{\alpha} \wedge U_{\beta} \mathrm{d} x^{\beta}\right]}{2} \\
& =\sum_{\alpha<\beta}\left(\partial_{\beta} U_{\alpha}-\partial_{\alpha} U_{\beta}+\left[U_{\alpha}, U_{\beta}\right]\right) \mathrm{d} x^{\beta} \wedge \mathrm{d} x^{\alpha},
\end{aligned}
$$

for all $\alpha, \beta \in \overline{1, m}$ and $\lambda \in \Lambda$. Hence, the above expression vanishes for a certain $u(x)$ if and only if (5.3) holds.

Example 5.1 The complex projective space $\mathbb{C} P^{1}$ can be understood via the Hopf fibration $h: S^{3} \rightarrow \mathbb{C} P^{1} \simeq S^{3} / S^{1} \simeq S^{2}$ as the space of orbits in the three-dimensional unit sphere $S^{3} \subset \mathbb{C}^{2}$ (relative to the canonical Hermitian product in $\mathbb{C}^{2}$ ) with respect to the natural action of $S^{1}$, understood as the Lie group of complex numbers with module one, on $S^{3}$ (see $[36,61]$ for details). Equivalently, $\mathbb{C} P^{1}$ amounts to the space of rankone Hermitian projectors $P$ onto $\mathbb{C}^{2}$, namely $P^{\dagger}=P, P^{2}=P$ and $\operatorname{tr} P=1[19,24]$. The solutions for the $\mathbb{C} P^{1}$ sigma model are given by mappings $P: S^{2} \rightarrow \mathbb{C} P^{1}$ where $S^{2}$ is understood as the compactification of $\mathbb{C}$ by gluing the infinity point topologically. The differential equations for the $\mathbb{C} P^{1}$ sigma model are given by

$\left[\partial_{+} \partial_{-} P(x, y), P(x, y)\right]=\frac{1}{4}\left[\nabla^{2} P, P\right]=0, \quad \nabla^{2}:=\partial_{x}^{2}+\partial_{y}^{2}, \quad \partial_{ \pm}:=\frac{1}{2}\left(\partial_{x} \pm \mathrm{i} \partial_{y}\right)$,

where $[\cdot, \cdot \cdot]$ stands for the commutator of operators. Hence, the $\mathbb{C} P^{1}$ sigma model can be understood as a system of PDEs on the jet bundle $J^{2}$ relative to the projection onto the second factor $\pi: \mathbb{C} P^{1} \times S^{2} \rightarrow S^{2}$. This system admits a LSP problem with a purely imaginary spectral parameter $\lambda \in \mathbb{C}$. To clearly show the geometric properties of this LSP, it is convenient to introduce the new variable $\theta:=\mathrm{i}\left(P-\mathrm{Id}_{2} / 2\right) \in \mathfrak{s u}(2)$. Then, the LSP reads

$$
\left\{\begin{array}{l}
\partial_{x} \Phi=U_{x}(\theta(x, y), \lambda) \Phi \\
\partial_{y} \Phi=U_{y}(\theta(x, y), \lambda) \Phi
\end{array}, \quad \Phi \in S U(2), \quad \bar{\lambda}=-\lambda\right.
$$


where

$$
\left\{\begin{aligned}
U_{x}(\theta(x, y), \lambda) & :=\frac{-2}{1-\lambda^{2}}\left(\left[\partial_{x} \theta(x, y), \theta(x, y)\right]-i \lambda\left[\partial_{y} \theta(x, y), \theta(x, y)\right]\right) \in \mathfrak{s u}(2), \\
U_{y}(\theta(x, y), \lambda) & :=\frac{-2}{1-\lambda^{2}}\left(\left[\partial_{x} \theta(x, y), \theta(x, y)\right] \mathrm{i} \lambda+\left[\partial_{y} \theta(x, y), \theta(x, y)\right]\right) \in \mathfrak{s u}(2) .
\end{aligned}\right.
$$

This leads to a real parametrized $\mathfrak{s u}(2)$-valued differential one-form on $\mathbb{C}$ given by

$$
\omega_{C P}:=U_{x}(\theta(x, y), \lambda) \mathrm{d} x+U_{y}(\theta(x, y), \lambda) \mathrm{d} y .
$$

Note that $\theta(x, y), \partial_{x} \theta(x, y), \partial_{y} \theta(x, y)$ are understood as particular curves in $\mathfrak{s u}(2)$.

The LSP of the $\mathbb{C} P^{1}$ model, its parametrized $\mathfrak{s u}(2)$-valued differential one-form, and the corresponding system of PDEs can be described through jet bundles. This suggests to us the following geometric approach to LSP equations and related structures based on jets and spectral differential forms taking values in $\mathfrak{s u}(2)$.

The system of PDEs (5.1) can be extended to $\mathcal{E}^{\infty} \subset J^{\infty}$ by assuming $[u] \in J^{\infty}$ and adding the conditions $D_{J}\left[\nabla^{2} P, P\right]=0$ for an arbitrary multi-index $J$. This allows us to define a new submanifold $\mathcal{E}^{\infty} \subset J^{\infty}$ where all previous functions on $J^{\infty}$ vanish. Then, the total derivatives $D_{x}, D_{y}$ become tangent to $\mathcal{E}^{\infty}$.

Let us develop a new formalism of the LSP problem via jets. Essentially, this is a reinterpretation of the algebraic approach given in [21] in terms of functions on rings and an improvement of the method on spaces of jets detailed in [26].

Consider that the hereafter called jet LSP for the differential equation (5.1) is a system of PDEs on $J^{\infty}$ of the form

$$
D_{\alpha} \Phi([u], \lambda)=U_{\alpha}([u], \lambda) \Phi([u], \lambda), \quad \alpha \in \overline{1, m}, \quad \Phi([u], \lambda) \in G
$$

The system of PDEs (5.6) must be involutive to be integrable, i.e.

$$
D_{\alpha} D_{\beta} \Phi([u], \lambda)=D_{\beta} D_{\alpha} \Phi([u], \lambda)
$$

Nevertheless, the unknown of this system of PDEs is defined on an infinite-dimensional jet space $J^{\infty}$. Hence, it is not obvious that this condition is sufficient to ensure integrability. Nevertheless, it can be proved under quite general conditions, e.g. the system must be analytic [42], that involutivity ensures integrability.

The following theorem enables us to extend the notion of integrability on finitedimensional manifolds to $J^{\infty}$ and the diffiety $\mathcal{E}^{\infty}$.

Theorem 5.1 The differential equation (5.6) has a solution $\Phi([u], \lambda)$, with $[u] \in \mathcal{E}^{\infty}$, if and only if $\mathbf{d}_{\omega} \omega=0$ on $\mathcal{E}^{\infty}$.

Proof Let us prove the direct part. If (5.6) has a solution on $\mathcal{E}^{\infty}$, then

$$
D_{\beta} D_{\alpha} \Phi=D_{\alpha} D_{\beta} \Phi, \quad \forall \lambda \in \Lambda, \forall \alpha, \beta \in \overline{1, m}
$$


implies that

$$
D_{\beta} U_{\alpha}-D_{\alpha} U_{\beta}+\left[U_{\alpha}, U_{\beta}\right]=0, \quad \forall \lambda \in \Lambda,
$$

on $\mathcal{E}^{\infty}$. Then,

$$
\mathbf{d}_{\omega} \omega=\sum_{\alpha<\beta}\left(D_{\beta} U_{\alpha}-D_{\alpha} U_{\beta}+\left[U_{\alpha}, U_{\beta}\right]\right) \mathrm{d} x^{\alpha} \wedge \mathrm{d} x^{\beta}=0, \quad \forall \lambda \in \Lambda
$$

on $\mathcal{E}^{\infty}$, whereupon the direct part follows. The converse stems from using that involutivity and analyticity of (5.6) on $\mathcal{E}^{\infty}$ gives rise to a general solution (cf. [42]).

It is worth noting that the pull-back by an arbitrary section $s(x):=(x, u(x))$ of $J^{0}$ of the structures related to spectral differential forms permit us to recover the formalism for parametrized $\mathfrak{g}$-valued differential forms. In particular, $j^{\infty} s^{*} \mathbf{d}_{\omega} \omega=0$ retrieves (5.3). Moreover, $j^{\infty} s^{*} \Phi([u], \lambda)$ provides a solution for the pull-back of the system (5.2), namely

$j^{\infty} s^{*} D_{\alpha} \Phi([u], \lambda)=j^{\infty} s^{*}[U([u], \lambda) \Phi([u], \lambda)] \Leftrightarrow \partial_{\alpha} \Phi(u(x), \lambda)=U_{\alpha}(u(x), \lambda) \Phi(u(x), \lambda)$.

In other words, the infinite-dimensional jet approach and spectral differential forms provide a method of representing the standard formalism for LSP when the structures appearing can be defined on infinite-dimensional jet manifolds.

Example 5.2 The $\mathbb{C} P^{1}$ sigma model can be naturally considered as a differential equation on $J^{2}$ of the form

$$
\left[D_{x}^{2} \theta+D_{y}^{2} \theta, \theta\right]=0
$$

This gives as a submanifold $\mathcal{E}_{s m} \subset J^{2}$. If the additional conditions are assumed

$$
D_{x}^{p} D_{y}^{q}\left[D_{x}^{2} \theta+D_{y}^{2} \theta, \theta\right]=0, \quad \forall p, q \in \overline{\mathbb{N}},
$$

then a submanifold $\mathcal{E}_{s m}^{\infty} \subset J^{\infty}$ is obtained and the derivatives $D_{x}, D_{y}$ are tangent to it. To describe (5.8), a jet LSP on $J^{\infty}$ is introduced:

$$
\left\{\begin{array}{l}
D_{x} \Phi([\theta], \lambda)=U_{x}([\theta], \lambda) \Phi([\theta], \lambda) \\
D_{y} \Phi([\theta], \lambda)=U_{y}([\theta], \lambda) \Phi([\theta], \lambda)
\end{array}, \quad \Phi([\theta], \lambda) \in S U(2), \quad \bar{\lambda}=-\lambda,\right.
$$

where

$$
\left\{\begin{aligned}
& U_{x}([\theta], \lambda):=\frac{-2}{1-\lambda^{2}}\left(\left[D_{x} \theta, \theta\right]-\mathrm{i} \lambda\left[D_{y} \theta, \theta\right]\right) \in \mathfrak{s u}(2), \\
& U_{y}([\theta], \lambda):=\frac{-2}{1-\lambda^{2}}\left(\left[D_{x} \theta, \theta\right] \mathrm{i} \lambda+\left[D_{y} \theta, \theta\right]\right) \in \mathfrak{s u}(2) .
\end{aligned}\right.
$$

The corresponding spectral differential one-form on $J^{\infty}$ is given by

$$
\omega([\theta], \lambda):=U_{x}([\theta], \lambda) \mathrm{d} x+U_{y}([\theta], \lambda) \mathrm{d} y .
$$


Hence

$$
\begin{aligned}
\mathbf{d}_{\omega} \omega & =D_{\beta} U_{\alpha} \otimes \mathrm{d} x^{\beta} \wedge \mathrm{d} x^{\alpha}-\frac{1}{2}\left[\left(U_{\alpha} \mathrm{d} x^{\alpha}\right) \wedge\left(U_{\alpha} \mathrm{d} x^{\alpha}\right)\right] \\
& =\left(D_{y} U_{x}-D_{x} U_{y}+\left[U_{x}, U_{y}\right]\right) \mathrm{d} y \wedge \mathrm{d} x \\
& =\left[\left(D_{x}^{2}+D_{y}^{2}\right) \theta, \theta\right] \mathrm{d} y \wedge \mathrm{d} x .
\end{aligned}
$$

Composing this with the infinite-prolongation $j^{\infty} s$ of a section $s(x, y):=(x, y$, $\theta(x, y))$ related to an arbitrary $\theta(x, y)$, we obtain that $j^{\infty} s^{*} \mathbf{d}_{\omega} \omega=0$ amounts to

$$
\begin{aligned}
0 & =j^{\infty} s^{*} \mathbf{d}_{\omega} \omega \\
& =\left(\partial_{y} U_{x}-\partial_{x} U_{y}+\left[U_{x}, U_{y}\right]\right) \mathrm{d} y \wedge \mathrm{d} x \\
& =\left(\partial_{y} U_{x}(\theta(t), \lambda)-\partial_{x} U_{y}(\theta(t), \lambda)+\left[U_{x}(\theta(t), \lambda), U_{y}(\theta(t), \lambda)\right]\right) \mathrm{d} y \wedge \mathrm{d} x .
\end{aligned}
$$

Finally, let us consider an assumption that will be useful in the following sections and appears in different practical applications of immersion formulas [27].

Let $\operatorname{Id}_{N}$ be an $N \times N$ identity matrix. Subsequently, we assume that there is an on-shell solution $\Phi([u], \lambda)$ to (5.2) satisfying the so-called on-shell condition

$$
\lim _{\lambda \rightarrow \infty} \Phi([u], \lambda)=\operatorname{Id}_{N} \in G, \quad \forall[u] \in \mathcal{E}^{\infty} .
$$

Example 5.3 A solution to the jet LSP for the $\mathbb{C} P^{1}$ sigma model reads (cf. [27])

$$
\Phi([\theta], \lambda)=\operatorname{Id}_{2}+\left(\frac{4 \lambda}{(1-\lambda)^{2}}-\frac{2}{1-\lambda}\right)\left(\frac{\mathrm{Id}_{2}}{2}-\mathrm{i} \theta\right)=\frac{\lambda(\lambda+1) \mathrm{Id}_{2}-\mathrm{i}(6 \lambda-2) \theta}{(\lambda-1)^{2}} \in S U(2),
$$

for $[u] \in \mathcal{E}^{\infty}, \lambda:=\mathrm{i} t$, and $t \in \mathbb{R}$. When $\lambda \rightarrow+\infty$, the above solution satisfies the on-shell condition.

\section{Immersion formulas}

Now we are interested in looking for a simultaneous infinitesimal deformation of the LSP and the zero curvature condition. This will be done in a geometric manner, which will recover the case of immersed soliton surfaces as a particular instance, and it will enable us to generalize the procedure to any immersed submanifold in Lie algebras. Our formalism will use spectral differential forms, which is very practical in applications. The formalism for parametrized $\mathfrak{g}$-valued differential forms can be easily obtained by considering the pull-back of given expressions for particular sections $s \in \Gamma(\pi)$ and/or their prolongations.

Subsequently, the dependence of all structures on the corresponding variables will be omitted to simplify the notation. We recall that $G$ and $\mathfrak{g}$ are assumed to be finitedimensional matrix Lie groups and Lie algebras respectively.

Consider a deformation

$$
\omega_{\epsilon}:=\omega+\epsilon \Upsilon, \quad \Phi_{\epsilon}:=R_{\exp (\epsilon F)} \Phi, \quad \forall \epsilon \in \mathbb{R}, \forall \lambda \in \Lambda,
$$


where $\omega, \Upsilon \in \Omega_{\lambda}^{1}\left(\mathcal{E}^{\infty}\right) \otimes \mathfrak{g}, \Phi \in C_{\lambda}^{\infty}\left(\mathcal{E}^{\infty}\right) \otimes G$ and $F \in C_{\lambda}^{\infty}\left(\mathcal{E}^{\infty}\right) \otimes \mathfrak{g}$ satisfy

$$
\mathbf{d}_{\omega_{\epsilon}} \omega_{\epsilon}=0, \quad R_{\Phi_{\epsilon}^{-1}} \mathbf{d} \Phi_{\epsilon}=\omega_{\epsilon}, \quad \forall \epsilon \in \mathbb{R}, \forall \lambda \in \Lambda
$$

Although the above spectral differential forms may admit extensions to $\Omega_{\lambda}\left(J^{\infty}\right) \otimes \mathfrak{g}$, e.g. the above $\omega$ is given, in applications, by the restriction to $\mathcal{E}^{\infty}$ of the spectral differential form related to a LSP, we will be mainly concerned with their values on-shell. The first condition in (6.1) implies that

$$
\mathbf{d}_{\omega_{\epsilon}} \omega_{\epsilon}=\mathbf{d} \omega_{\epsilon}-\frac{1}{2}\left[\omega_{\epsilon} \wedge \omega_{\epsilon}\right]=0, \quad \forall \epsilon \in \mathbb{R}, \forall \lambda \in \Lambda
$$

The latter is satisfied if and only if $\mathbf{d}_{\omega} \omega=0$ on-shell and one has that

$$
0=\left.\frac{\mathrm{d}}{\mathrm{d} \epsilon}\right|_{\epsilon=0}\left(\mathbf{d} \omega_{\epsilon}-\frac{1}{2}\left[\omega_{\epsilon} \wedge \omega_{\epsilon}\right]\right)=\mathbf{d} \Upsilon-[\omega \wedge \Upsilon]=\mathbf{d}_{2} \omega \Upsilon
$$

where $\Upsilon:=A_{\alpha} \mathrm{d} x^{\alpha}$ in coordinates. Under previous conditions, it can be proved that $\mathbf{d} F=\operatorname{Ad}_{\Phi^{-1}} \Upsilon$ for a certain $F \in C_{\lambda}^{\infty}\left(\mathcal{E}^{\infty}\right) \otimes \mathfrak{g}$ (cf. [26,27]). The main task now is to provide methods to obtain $F$ from a certain $\mathbf{d}_{2 \omega}$-closed $\Upsilon$.

Since our deformation does not transform $\lambda$, the singularity structure of the $\omega_{\epsilon}$ in the spectral parameter $\lambda$ remains untouched. Each $\omega$ and $\Upsilon$ satisfying the above equations generate a submanifold immersed in the Lie algebra $\mathfrak{g}$ as explained next in this section. It is worth noting that our geometric approach allows us to simplify previous proofs in the literature (see for instance [20,26,27]). Moreover, our proof is general for any arbitrary $M$ and deals not only with $M=\mathbb{R}^{2}$ as is standard in the literature [10,12,18,20,26,27].

We then say that $\Phi$ is an on-shell spectral solution of the LSP problem when $\Phi([u], \lambda)$ is a particular solution of (5.6) for every $\lambda$ and every $[u] \in \mathcal{E}^{\infty}$. We may assume that $\Phi$ is extended to other $[u]$ out of $\mathcal{E}^{\infty}$ but in that case we cannot ensure that $\Phi([u], \lambda)$ will be a solution of (5.6) for all values of $\lambda \in \Lambda$. In such a case, we say that $\Phi([u], \lambda)$ is an off-shell spectral solution of (2.3).

Lemma 6.1 Let $\Phi$ be an on-shell solution of the jet LSP (5.6) and let $\omega$ be the associated element in $\Omega_{\lambda}^{1}\left(J^{\infty}\right) \otimes \mathfrak{g}$ satisfying $\mathbf{d}_{\omega} \omega=0$ for all $\lambda \in \Lambda$ on-shell. Then,

$$
\mathbf{d} \operatorname{Ad}_{\Phi^{-1}} \vartheta=\operatorname{Ad}_{\Phi^{-1}} \mathbf{d}_{2 \omega} \vartheta, \quad \forall \vartheta \in \Omega_{\lambda}\left(\mathcal{E}^{\infty}\right) \otimes \mathfrak{g}
$$

Proof Let us prove (6.2) for a spectral differential k-form, say $\vartheta:=f_{J}^{j} \mathrm{~d} x^{J} \otimes v_{j} \in$ $\Omega_{\lambda}^{k}\left(\mathcal{E}^{\infty}\right) \otimes \mathfrak{g}$, where $v_{1}, \ldots, v_{r}$ stands for a basis of $\mathfrak{g}$. The validity of equality (6.2) on the whole $\Omega_{\lambda}\left(\mathcal{E}^{\infty}\right) \otimes \mathfrak{g}$ follows from this by linearity. Now,

$$
\mathbf{d A d}_{\Phi^{-1}} \vartheta=\left(D_{\alpha} f_{J}^{j}\right) \mathrm{d} x^{\alpha} \wedge \mathrm{d} x^{J} \otimes \operatorname{Ad}_{\Phi^{-1}}\left(v_{j}\right)+f_{J}^{j} \mathrm{~d} x^{\alpha} \wedge \mathrm{d} x^{J} \otimes D_{\alpha}\left(\operatorname{Ad}_{\Phi^{-1}}\left(v_{j}\right)\right)
$$


Since $D_{\alpha} \Phi^{-1} \mathrm{~d} x^{\alpha}=-L_{\Phi^{-1}} \omega$ on-shell and writing $\omega=\mathrm{d} x^{\alpha} \otimes U_{\alpha}$, it follows that $D_{\alpha} \operatorname{Ad}_{\Phi^{-1}}\left(v_{j}\right)=-\operatorname{Ad}_{\Phi^{-1}}\left(\left[U_{\alpha}, v_{j}\right]\right)$ and

$$
\mathbf{d A d}_{\Phi^{-1}} \vartheta=\operatorname{Ad}_{\Phi^{-1}}(\mathbf{d} \vartheta-[\omega \wedge \vartheta])=\operatorname{Ad}_{\Phi^{-1}} \mathbf{d}_{2 \omega} \vartheta
$$

Lemma 6.2 Let $\Phi$ be an on-shell spectral solution of the jet LSP (5.6) and let $\omega \in \Omega_{\lambda}^{1}\left(J^{\infty}\right) \otimes \mathfrak{g}$ be its associated spectral differential one-form. If $\Upsilon$ is a spectral differential one-form on-shell, then there is a locally defined $F \in C_{\lambda}^{\infty}\left(\mathcal{E}^{\infty}\right) \otimes \mathfrak{g}$ such that

$$
\mathbf{d} F=\operatorname{Ad}_{\Phi^{-1}} \Upsilon
$$

if and only if $\mathbf{d}_{2 \omega} \Upsilon=0$ on-shell.

Proof From Lemma 6.1 and the present assumptions, it follows that

$$
\mathbf{d A d}_{\Phi^{-1}} \Upsilon=\operatorname{Ad}_{\Phi^{-1}} \mathbf{d}_{2 \omega} \Upsilon
$$

on-shell. Hence, $\operatorname{Ad}_{\Phi^{-1}} \Upsilon$ is $\mathbf{d}$-closed (on-shell) if and only if $\mathbf{d}_{2 \omega} \Upsilon=0$ on-shell. From the properties of the cohomology $\mathbf{d}$ on $\Omega_{\lambda}\left(\mathcal{E}^{\infty}\right) \otimes \mathfrak{g}$, the spectral differential 1form on-shell $\operatorname{Ad}_{\Phi^{-1}} \Upsilon$ is closed if and only if there exists locally an $F \in C_{\lambda}^{\infty}\left(\mathcal{E}^{\infty}\right) \otimes \mathfrak{g}$ such that (6.3) is satisfied. This finishes the proof.

To fix accurately the notation of our paper, we introduce the following notion.

Definition 6.3 We call $F$ in (6.3) an immersion formula for the jet LSP (5.6).

Lemma 6.2 shows the interest of finding the on-shell spectral differential oneforms $\Upsilon$ satisfying the equation $\mathbf{d}_{2 \omega} \Upsilon=0$ on-shell for a certain $\omega \in \Omega_{\lambda}^{1}\left(J^{\infty}\right) \otimes$ $\mathfrak{g}$ satisfying the condition $\mathbf{d}_{\omega} \omega=0$ on-shell. There are certain known methods to obtain $\Upsilon$. The work [39] employs an exact differential one-form, the generalized Weierstrass embedding formula, to obtain an immersion formula. The articles [10,58] show that the admissible symmetries of the ZCC (5.3) when $m=2$ include a conformal transformation of the spectral parameter $\lambda$, a gauge transformation of the wavefunction $\Phi$ in the LSP (5.2) and generalized symmetries of the integrable system (5.1). All these symmetries can be used to determine explicitly an immersion formula $F$. Most works deal with applications where the image of $F$ is a two-dimensional surface. Less commonly applications deal with an $F$ whose image is an immersed submanifold in a Lie algebra $[3,11]$. All such results can be found in a simpler and more general way, e.g. allowing the image of $F$ to be a general immersed submanifold in $\mathfrak{g}$, by using the following theorem.

Theorem 6.4 Let $\Phi$ be an on-shell spectral solution of the associated LSP (5.6) and let $\omega \in \Omega_{\lambda}^{1}\left(J^{\infty}\right) \otimes \mathfrak{g}$ be the associated spectral differential form. Assume that $\Upsilon \in$ $\Omega_{\lambda}^{1}\left(\mathcal{E}^{\infty}\right) \otimes \mathfrak{g}$ takes the form on-shell

$$
\Upsilon:=\beta(\lambda) \partial_{\lambda} \omega+\mathbf{d}_{2 \omega} S+\mathcal{L}_{\mathrm{pr} X_{R}} \omega
$$


Here, $\beta(\lambda)$ is an arbitrary scalar function depending only on $\lambda$, the function $S$ is an arbitrary element of $C_{\lambda}^{\infty}\left(\mathcal{E}^{\infty}\right) \otimes \mathfrak{g}$, and the vector field $X_{R}:=R^{i} \partial_{u^{i}}$ is a generalized symmetry of $\Delta\left[j_{x}^{p} u\right]=0$. Then, there exists an immersion formula $F \in C_{\lambda}^{\infty}\left(\mathcal{E}^{\infty}\right) \otimes \mathfrak{g}$ given by the formula

$$
F=\beta(\lambda) L_{\Phi^{-1}} \partial_{\lambda} \Phi+\operatorname{Ad}_{\Phi^{-1}} S+R_{\Phi^{-1}} \operatorname{pr} X_{R} \Phi
$$

Proof Due to historical reasons, let us prove that the three terms

$$
\Upsilon^{S T}:=\beta(\lambda) \partial_{\lambda} \omega, \quad \Upsilon^{C D}:=\mathbf{d}_{2 \omega} S, \quad \Upsilon^{F G}:=\mathcal{L}_{\operatorname{pr} X_{R}} \omega
$$

of the spectral $\mathfrak{g}$-valued differential one-form on-shell (6.4) are such that the action of $\operatorname{Ad}_{\Phi^{-1}}$ on each one is the differential (relative to $\mathbf{d}$ ) of the $\mathfrak{g}$-valued spectral functions on-shell

$$
F^{S T}:=\beta(\lambda) L_{\Phi^{-1}} \partial_{\lambda} \Phi, \quad F^{C D}:=\operatorname{Ad}_{\Phi^{-1}} S, \quad F^{F G}:=L_{\Phi^{-1}} \operatorname{pr} X_{R} \Phi,
$$

respectively. This proves that the latter $\mathfrak{g}$-valued spectral functions are immersion formulas for (6.4).

Let us start by studying the Sym-Tafel spectral differential 1-form, namely $\Upsilon^{S T}$. Using the fact that $\Phi$ is an on-shell spectral solution of (5.2), we obtain on-shell

$$
\begin{aligned}
\mathbf{d} F^{S T} & =D_{\alpha}\left(F^{S T}\right) \mathrm{d} x^{\alpha} \\
& =\beta(\lambda)\left[\left(D_{\alpha} \Phi^{-1}\right) \partial_{\lambda} \Phi+\Phi^{-1} D_{\alpha} \partial_{\lambda} \Phi\right] \mathrm{d} x^{\alpha} \\
& =\beta(\lambda)\left[-\Phi^{-1} U_{\alpha} \partial_{\lambda} \Phi+\Phi^{-1} \partial_{\lambda}\left(U_{\alpha} \Phi\right)\right] \mathrm{d} x^{\alpha} \\
& =\beta(\lambda) \operatorname{Ad}_{\Phi^{-1}} \partial_{\lambda} \omega \\
& =\operatorname{Ad}_{\Phi^{-1}} \Upsilon^{S T} .
\end{aligned}
$$

Let us now study the Cieśliński-Doliwa 1-form, i.e. $\Upsilon^{C D}=\mathbf{d}_{2 \omega} S$. From Lemma 6.1 , it follows that

$$
\mathbf{d} F^{C D}=\mathbf{d A d}_{\Phi^{-1}} S=\operatorname{Ad}_{\Phi^{-1}} \mathbf{d}_{2 \omega} S=\operatorname{Ad}_{\Phi^{-1}} \Upsilon^{C D} .
$$

The Fokas-Gel'fand 1-form, i.e. $\Upsilon^{F G}=\mathcal{L}_{\mathrm{pr} X_{R}} \omega$, induces a function $F^{F G} \in$ $C_{\lambda}^{\infty}\left(\mathcal{E}^{\infty}\right) \otimes \mathfrak{g}$. To show this it is enough to observe that since $\left[D_{\alpha}, \operatorname{pr} X_{R}\right]=0$ one has on-shell that

$$
\begin{aligned}
\mathbf{d} F^{F G} & =\left(-\Phi^{-1} U_{\alpha} \operatorname{pr} X_{R} \Phi+L_{\Phi^{-1}} \operatorname{pr} X_{R}\left(U_{\alpha} \Phi\right)\right) \mathrm{d} x^{\alpha} \\
& =\operatorname{Ad}_{\Phi^{-1}} \Upsilon^{F G} .
\end{aligned}
$$




\section{Relations between immersion formulas}

The work [27] showed that there is a gauge transformation mapping the Sym-Tafel and Cieśliński-Doliwa immersion formulas to each other when the potential, $S$, of one of them is invertible. This result can be easily proved through our techniques.

Proposition 7.1 A vector field $D_{\lambda}$ is a symmetry of the ZCC condition $\mathbf{d}_{\omega} \omega=0$ associated with $\Delta\left(j_{x}^{p} u\right)=0$ if and only if there exists an $S^{S T} \in C_{\lambda}^{\infty}\left(\mathcal{E}^{\infty}\right) \otimes \mathfrak{g}$ such that on-shell

$$
\mathbf{d}_{2 \omega} S^{S T}=\beta(\lambda) \mathcal{L}_{D_{\lambda}} \omega .
$$

Proof Lemma 6.2 establishes that $\operatorname{Ad}_{\Phi^{-1}} \Upsilon=\mathbf{d} F$ locally for a certain $F \in C_{\lambda}^{\infty}\left(\mathcal{E}^{\infty}\right) \otimes$ $\mathfrak{g}$ if and only if $\mathbf{d}_{2 \omega} \Upsilon=0$ on-shell. Since $\left[\mathbf{d}, \mathcal{L}_{D_{\lambda}}\right]=0$ off-shell, one has

$$
\begin{aligned}
\mathbf{d}_{2 \omega}\left[\beta(\lambda) \mathcal{L}_{D_{\lambda}} \omega\right] & =\beta(\lambda)\left[\mathbf{d} \mathcal{L}_{D_{\lambda}} \omega-\left[\omega \wedge \mathcal{L}_{D_{\lambda}} \omega\right]\right] \\
& =\beta(\lambda)\left[\mathcal{L}_{D_{\lambda}} \mathbf{d} \omega-\frac{1}{2} \mathcal{L}_{D_{\lambda}}[\omega \wedge \omega]\right]=\beta(\lambda) \mathcal{L}_{D_{\lambda}} \mathbf{d}_{\omega} \omega .
\end{aligned}
$$

It is worth noting that although $\mathbf{d}_{\omega} \omega=0$ on-shell, $D_{\lambda} \mathbf{d}_{\omega} \omega$ may not vanish, as these terms give the infinitesimal variation of $\mathbf{d}_{\omega} \omega$ along a one-parametric family of curves $\left(u_{\epsilon}(t), \lambda_{\epsilon}\right)$ generated by the flow of $D_{\lambda}$. Only $u_{0}(t)$ must be a solution to $\Delta\left(j_{x}^{p} u\right)=0$, while $\mathbf{d}_{\omega} \omega$ may not vanish on $\left(u_{\epsilon}(x), \lambda_{\epsilon}\right)$.

If $\beta(\lambda) \mathcal{L}_{D_{\lambda}} \omega=\mathbf{d} S^{S T}$ on-shell, then (7.2) vanishes and $\mathcal{L}_{D_{\lambda}} \mathbf{d}_{\omega} \omega=0$ on-shell, i.e. $D_{\lambda}$ is symmetry of the ZCC condition. Conversely, if $D_{\lambda}$ is a symmetry of ZCC condition for the $\Delta\left(j_{x}^{p} u\right)$, then the expression (7.2) vanishes on-shell and, due to the properties of the $\mathbf{d}_{2 \omega}$ cohomology, there exists a $S^{S T} \in C_{\lambda}^{\infty}\left(\mathcal{E}^{\infty}\right) \otimes \mathfrak{g}$ such that $\mathbf{d} S^{S T}=\beta(\lambda) \mathcal{L}_{D_{\lambda}} \omega$ on-shell.

It is worth noting that the cohomological proof of this theorem is almost trivial. Meanwhile, its counterpart in coordinates in [27] is much longer and obscure.

Analogously, it is possible to see that the remaining terms within the $\mathfrak{g}$-valued spectral differential one-form $\Upsilon$ in Theorem 6.4 are induced from other types of gauges. Then, these gauges allow us to map one term into another.

Proposition 7.2 A vector field $X_{R}$ is a generalized symmetry of the ZCC $\mathbf{d}_{\omega} \omega$ of the jet LSP associated with an integrable system $\Delta\left[j_{x}^{p} u\right]=0$ if and only if there exists a spectral function $S^{F G} \in C_{\lambda}^{\infty}\left(\mathcal{E}^{\infty}\right) \otimes \mathfrak{g}$ such that on-shell

$$
\mathbf{d}_{2 \omega} S^{F G}=\mathcal{L}_{\mathrm{pr} X_{R}} \omega .
$$

Proof Using the Lemma 6.1, we obtain that $\mathbf{d}_{2 \omega} \Upsilon^{F G}=\operatorname{Ad}_{\Phi} \mathbf{d A d}_{\Phi^{-1}} \Upsilon^{F G}$. A short computation shows that

$$
\begin{aligned}
\mathbf{d A d}_{\Phi^{-1}} \Upsilon F \mathrm{~F} & =D_{\beta}\left[\Phi^{-1}\left(\mathcal{L}_{\mathrm{pr}_{X_{R}}} D_{\alpha} \Phi\right)-\Phi^{-1} U_{\alpha}\left(\mathcal{L}_{\mathrm{pr}_{X_{R}}} \Phi\right)\right] \mathrm{d} x^{\beta} \wedge \mathrm{d} x^{\alpha} \\
& =\left(\Phi^{-1} \mathcal{L}_{\operatorname{pr}_{X_{R}}} D_{\beta} D_{\alpha} \Phi+\Phi^{-1} U_{\beta} U_{\alpha} \mathcal{L}_{\mathrm{pr}_{X_{R}}} \Phi-\Phi^{-1} D_{\beta} U_{\alpha} \mathcal{L}_{\mathrm{pr}_{X_{R}}} \Phi\right) \mathrm{d} x^{\beta} \wedge \mathrm{d} x^{\alpha} \\
& =\sum_{\alpha<\beta}\left(\Phi^{-1} \mathcal{L}_{\operatorname{pr}_{X_{R}}}\left(D_{\beta} D_{\alpha}-D_{\alpha} D_{\beta}\right) \Phi-\Phi^{-1}\left(\mathbf{d}_{\omega} \omega\right) \mathcal{L}_{\operatorname{pr}_{X_{R}}} \Phi\right) \mathrm{d} x^{\beta} \wedge \mathrm{d} x^{\alpha} \\
& =\Phi^{-1} \mathcal{L}_{\operatorname{pr}_{X_{R}}}\left(\mathbf{d}_{\omega} \omega\right) .
\end{aligned}
$$


Fig. 1 Representation of the relations between the wavefunction $\Phi \in G$ and the $\mathfrak{g}$-valued ST and FG immersion formulas for soliton submanifolds

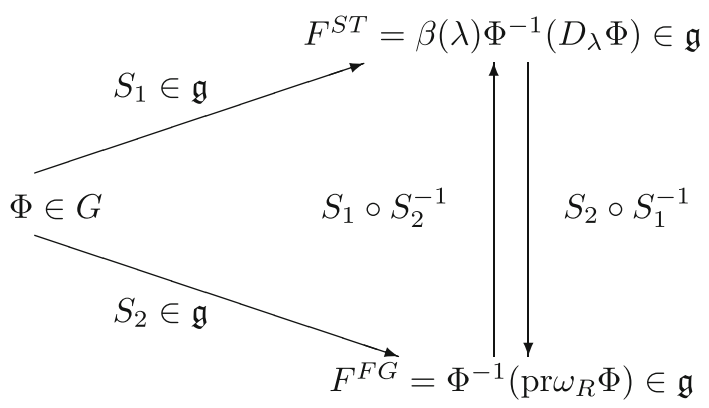

If $\Upsilon^{F G}=\mathbf{d}_{2 \omega} S^{F G}$ on-shell, then $\mathcal{L}_{\operatorname{pr}_{X}} \mathbf{d}_{\omega} \omega=0$ on-shell and the generalized symmetry $X_{R}$ is a symmetry of $\Delta\left(j_{x}^{p} u\right)$. Conversely, if $X_{R}$ is a generalized symmetry of $\Delta\left(j_{x}^{p} u\right)$, then $\mathbf{d}_{2 \omega} \Upsilon^{F G}=0$ and $\Upsilon^{F G}=\mathbf{d}_{2 \omega} S^{F G}$ on-shell for a certain $S^{F G} \in$ $C_{\lambda}^{\infty}\left(\mathcal{E}^{\infty}\right) \otimes \mathfrak{g}$.

Let us show how a way to map different immersion maps among themselves.

Corollary 7.3 Let $\Upsilon_{1}, \Upsilon_{2} \in \Omega_{\lambda}^{1}\left(\mathcal{E}^{\infty}\right) \otimes \mathfrak{g}$ be two solutions of $\mathbf{d}_{2 \omega} \Upsilon=0$ on-shell. Then, there exists an $F \in C_{\lambda}^{\infty}\left(\mathcal{E}^{\infty}\right) \otimes \operatorname{Aut}(\mathfrak{g})$, a so-called gauge transformation, mapping the immersion formula $S_{1}$ of $\Upsilon_{1}$ onto the immersion formula $S_{2}$ of $\Upsilon_{2}$ or vice versa, whenever $S_{1}$ or $S_{2}$ are invertible matrices of $\mathfrak{g}$, respectively.

Proof It is immediate that under the given assumptions, $F=S_{2} \circ S_{1}^{-1}$ or $F=S_{1} \circ S_{1}^{-2}$ is the required element for invertible $S_{1}$ or $S_{2}$, respectively.

In particular, the application of the above corollary for the Sym-Tafel and FokasGelf'and terms is summarised in the diagram described in Fig. 1.

\section{Immersion formulas for PDEs with initial conditions}

It frequently happens in applications that an LSP only describes a subspace of particular solutions of a system of PDEs. For instance, the ZCC of the LSP just recovers the particular solutions of the system of PDEs satisfying a particular initial condition. The aim of this section is to recover immersion formulas and other results in this case. To illustrate the problem under consideration, let us analyse the PDE in $J^{2}$ for $\pi:(u ; x, y) \in \mathbb{R} \times \mathbb{R}^{2} \mapsto(x, y) \in \mathbb{R}^{2}$ given by

$$
u_{x x}-\frac{1}{2} f^{\prime}(u)=0
$$

for some function $f: u \in \mathbb{R} \mapsto f(u) \in \mathbb{R}$. It is straightforward to prove that the function $u_{x}^{2}-f(u)$ is a constant of motion of (8.1) and this can be used to obtain the general solution to (8.1). Nevertheless, not every solution of our PDE 
satisfies $u_{x}^{2}=f(u)$, which can be understood as a boundary condition. For instance, if $f(u)=0$ the function $u(x, y)=x$ satisfies (8.1) without obeying the given initial condition. A rather simple calculation shows that the PDE (8.1), along with the previous initial condition, admits a Lax pair

$$
\partial_{x} \Phi=L \Phi, \quad \partial_{y} \Phi=K \Phi, \quad \Phi \in S L(2, \mathbb{R}), L, K \in \mathfrak{s l}(2, \mathbb{R}),
$$

where

$$
L:=\frac{1}{2}\left[\begin{array}{cc}
0 & \frac{f^{\prime}(u)}{u+\lambda}-\frac{f(u)-g(-\lambda)}{(u+\lambda)^{2}} \\
1 & 0
\end{array}\right], \quad K:=\frac{1}{2}\left[\begin{array}{cc}
u_{x} & -\frac{f(u)-g(-\lambda)}{u+\lambda} \\
u+\lambda & -u_{x}
\end{array}\right]
$$

and $g(-\lambda):=\operatorname{det}(K)$ is assumed to be a rational function. The system (8.1) admits the generalized Lie symmetries

$$
Q_{1}:=u_{x} \frac{\partial}{\partial u} \quad Q_{2}:=u_{x}\left(\int f^{-3 / 2}(u) d x\right) \frac{\partial}{\partial u} .
$$

Since pr $Q_{1}-D_{x}=\partial_{x}$, the vector field $Q_{1}$ induces a translation in the $x$ variable, which is a symmetry of the initial condition $u_{x}^{2}-f(u)=0$ and (8.1). Therefore, we can redefine our PDE by adding the initial condition. This enables us to apply all our previous results to this new problem.

On the other hand, a straightforward calculation shows that $Q_{2}$ is an infinitesimal symmetry only of (8.1), i.e. it does not leave invariant the initial condition. Consequently, the formalism given in the previous section does not apply to this new case. Despite this, it is possible to rewrite all previous results to deal with it.

Let us consider a system of PDEs given by (2.3) and a LSP which only applies to solutions of (2.3) with some additional particular conditions. Let the new system by described by $\mathcal{S}^{\prime} \subset J^{p}$. The parametrized $\mathfrak{g}$-valued differential forms of $\Omega_{\mathcal{S}}(M) \otimes \mathfrak{g}$ can be reduced to the set $\mathcal{S}^{\prime}$. The restrictions of Theorem 3.4 and Corollary 3.5 to $\mathcal{S}^{\prime}$ remain true. This allows us to restrict our cohomologies, $\mathbf{d}$ and $\mathbf{d}_{2 \omega}$ to $\Omega_{\mathcal{S}^{\prime}}(M) \otimes \mathfrak{g}$. Moreover, Lemmas 6.1 and 6.2 remain true when $\Phi$ is a solution of the LSP for curves $u(x)$ in $\mathcal{S}^{\prime}$.

Let us now comment on spectral differential forms. If we consider the initial condition as a part of the initial system of PDEs, the space of solutions of the new system of PDEs with the initial conditions induces a submanifold $\mathcal{E}_{2} \subset \mathcal{E} \subset J^{p}$ and a new prolongation $\mathcal{E}_{2}^{\infty}$. Hence, all previous results for $\mathcal{E}$ now apply to $\mathcal{E}_{2}$. Since they can be trivially restricted to the systems of PDEs on $\mathcal{E}_{2}$, the restriction of the CielinskiDoliwa or the Sym-Tafel immersion formulas remain valid for the restriction of the initial system.

In spite of previous arguments, there exists one important difference relative to previous immersion formulas in the case of the restriction. The Lie symmetries of $\mathcal{E}$ do not necessarily give rise to Lie symmetries of the associated LSP. Consequently, the FG immersion formula must be modified accordingly. This will be solved by adding a new term in the Fokas-Gelf' and one-form as done next. 
Theorem 8.1 Let $\Phi$ be an on-shell spectral solution of the associated LSP (5.2) defined over a submanifold of solutions of the initial systems of PDEs and let $\omega \in \Omega_{\lambda}^{1}\left(J^{\infty}\right) \otimes \mathfrak{g}$ be the associated $\mathfrak{g}$-valued spectral differential form. Assume that $\Upsilon^{F G^{\prime}} \in \Omega_{\lambda}^{1}\left(J^{\infty}\right) \otimes \mathfrak{g}$ takes the form on-shell

$$
\Upsilon^{F G^{\prime}}:=\mathcal{L}_{\operatorname{pr} X_{R}} \omega+R_{\Phi^{-1}} \operatorname{pr} X_{R}\left(D_{\alpha} \Phi-U_{\alpha} \Phi\right)
$$

Here, the vector field $X_{R}$ is a generalized symmetry of $\Delta\left(j_{x}^{p} u\right)=0$. Then,

$$
F^{F G^{\prime}}=L_{\Phi^{-1}} \operatorname{pr} X_{R} \Phi \in C_{\lambda}^{\infty}\left(\mathcal{E}_{2}^{\infty}\right) \otimes \mathfrak{g}
$$

Proof As in Theorem 6.4, let us prove that $\Upsilon^{F G^{\prime}}:=\mathcal{L}_{\operatorname{pr} X_{R}} \omega+R_{\Phi^{-1}} \operatorname{pr} X_{R}\left(D_{\alpha} \Phi-\right.$ $\left.U_{\alpha} \Phi\right) \mathrm{d} x^{\alpha}$, is such that the action of $\mathrm{Ad}_{\Phi^{-1}}$ on it gives the differential (relative to d) of the $\mathfrak{g}$-valued spectral function (8.3). To show this it is enough to recall that since $\left[D_{\alpha}, \operatorname{pr} X_{R}\right]=0$ and then

$$
\begin{aligned}
\mathbf{d} F^{F G^{\prime}} & =\left(-L_{\Phi^{-1}} U_{\alpha} \operatorname{pr} X_{R} \Phi+L_{\Phi^{-1}} \operatorname{pr} X_{R}\left(D_{\alpha} \Phi-U_{\alpha} \Phi\right)+L_{\Phi^{-1}} \operatorname{pr} X_{R}\left(U_{\alpha} \Phi\right)\right) \mathrm{d} x^{\alpha} \\
& =\operatorname{Ad}_{\Phi^{-1}}\left(\mathcal{L}_{\operatorname{pr} X_{R}} \omega+R_{\Phi^{-1}} \operatorname{pr} X_{R}\left(D_{\alpha} \Phi-U_{\alpha} \Phi\right)\right) \\
& =\operatorname{Ad}_{\Phi^{-1}} \Upsilon^{F G^{\prime}} .
\end{aligned}
$$

\section{Soliton surfaces for $\mathbb{C} P^{N-1}$ sigma models}

Let us illustrate our techniques by studying immersion formulas for the $\mathbb{C} P^{N-1}$ sigma model. Based on the Gram-Schmidt orthogonalization procedure, a method for constructing an entire class of solutions on $S^{2}$ admitting the finite action of the $\mathbb{C} P^{N-1}$ sigma model was proposed by Dim et al. [16,17] and later studied by Sasaki [56]. In particular, we focus on the case $N=3$, whose immersion formulas will allow us to study the so-called Veronese surfaces and the mixed type soliton surfaces $[2,6,66]$. A final type of induced surface will be also briefly commented on (for details on these models see $[2,35,45,65,66])$.

The study of integrable surfaces for $\mathbb{C} P^{N-1}$ models can be accomplished via rankone Hermitian projectors. A matrix $P(z, \bar{z})$, with $z \in \mathcal{R}:=\mathbb{C} \cup\{\infty\}$, is said to be a rank-one Hermitian projector if $P^{2}=P, P=P^{\dagger}, \operatorname{tr} P=1$. The image of $P$ is a complex one-dimensional space of $\mathbb{C}^{N}$. Therefore, there exists

$$
f: \mathcal{R} \supseteq \Omega \ni \xi:=x+i y \mapsto\left(f_{0}(\xi), f_{1}(\xi), \ldots, f_{N-1}(\xi)\right)^{T} \in \mathbb{C}^{N} \backslash\{0\}
$$

such that $P=\frac{f \otimes f^{\dagger}}{f^{\dagger} f}$. The equations of motion for the $\mathbb{C} P^{N-1}$ model read

$$
\left[\left(\partial_{x}^{2}+\partial_{y}^{2}\right) P, P\right]=0
$$


Geometrically, the above expression is given by the differential equation on the secondorder jet $J^{2}$ of the bundle $\pi:(\theta, \xi) \in \mathfrak{s u}(N) \times \mathcal{R} \mapsto \xi \in \mathcal{R}$ given by

$$
\left[\left(\partial_{x}^{2}+\partial_{y}^{2}\right) \theta, \theta\right]=0
$$

where $\theta:=\mathrm{i}\left(P-\operatorname{Id}_{N} / N\right) \in \mathfrak{s u}(N)$. This system of PDEs induces in the standard way a submanifold of $\mathcal{E} \subset J^{2}$ [51]. To apply the spectral differential form approach, (9.2) has to be extended to $J^{\infty}$ by adding, as said in Sect. 2, the conditions $D_{J}\left[\left(\partial_{1}^{2}+\partial_{2}^{2}\right) \theta, \theta\right]=$ 0 , for all multi-indexes $J$ [62]. Then, $\mathcal{E}^{\infty}$ reads (see [62])

$$
\left[\left(D_{x}^{2}+D_{y}^{2}\right) \theta, \theta\right]=0, \quad D_{J}\left[\left(\partial_{x}^{2}+\partial_{y}^{2}\right) \theta, \theta\right]=0, \quad \forall J
$$

The jet LSP associated with (9.3) is given by $[48,65]$

$$
\begin{aligned}
& D_{\alpha} \Phi=\hat{U}_{\alpha} \Phi, \quad \alpha \in\{x, y\}, \quad \lambda=i t, \quad t \in \mathbb{R}, \\
& \hat{U}_{1}:=\frac{-(1+i \lambda)}{1-\lambda^{2}}\left[\left(D_{x}+D_{y}\right) \theta, \theta\right], \quad \hat{U}_{2}:=\frac{1+i \lambda}{1-\lambda^{2}}\left[\left(D_{x}-D_{y}\right) \theta, \theta\right],
\end{aligned}
$$

with a soliton solution on-shell $\Phi=\Phi([\theta], \lambda) \in S U(N)$ which goes to $\operatorname{Id}_{N}$ as $\lambda \rightarrow \infty$ and with the spectral functions $\hat{U}_{x}([\theta], \lambda), \hat{U}_{y}([\theta, \lambda])$ taking values in $\mathfrak{s u}(N)$ $[65,66]$. This system is related to the $\mathfrak{s u}(N)$-valued spectral differential one-form

$$
\omega:=\mathrm{d} x \otimes \hat{U}_{x}+\mathrm{d} y \otimes \hat{U}_{y}
$$

In fact, the integrability condition for (9.4) reads as

$$
\left.\mathbf{d}_{\omega} \omega=\mathrm{d} x \wedge \mathrm{d} y\left[\left(D_{x}^{2}+D_{y}^{2}\right) \theta, \theta\right]\right)=0 .
$$

The above vanishes on a space larger than $\mathcal{E}^{\infty}$, but the solutions of this differential equation are exactly those of (9.3) and (9.1). Consequently, the spectral $\mathfrak{s u}(N)$-differential form (9.5) vanishes on-shell, i.e. on $\mathcal{E}^{\infty} \subset J^{\infty}$.

To study the immersion submanifolds for (9.2), it is interesting to obtain a spectral solution of (9.4). This can be done by adapting to $J^{\infty}$ the rising and lowering projectors for $\mathbb{C} P^{N-1}$ models [23, p. 187], namely

$$
\Pi_{-}(\theta):=\frac{\bar{D} \theta(\mathcal{F}-i \theta) D \theta}{\operatorname{tr}(\bar{D} \theta(\mathcal{F}-i \theta) D \theta)}, \quad \Pi_{+}(\theta):=\frac{D \theta(\mathcal{F}-i \theta) \bar{D} \theta}{\operatorname{tr}(D \theta(\mathcal{F}-i \theta) \bar{D} \theta)}, \quad \mathcal{F}:=\frac{1}{N} \mathrm{I}_{N},
$$

where the traces in the denominators are different from zero for $\theta \neq 0$ unless the whole matrix is zero and $D:=\left(D_{x}+i D_{y}\right) / 2$ and $\bar{D}:=\left(D_{x}-i D_{y}\right) / 2$. Then, a family of spectral solutions $\Phi_{k}([\theta], \lambda)$ to the jet LSP (9.4) read as (cf. [27])

$$
\Phi_{k}([\theta], \lambda):=\operatorname{Id}_{N}+\frac{4 \lambda}{(1-\lambda)^{2}} \sum_{j=0}^{k-1} \Pi_{-}^{j}(\theta)-\frac{2}{1-\lambda}\left(\frac{1}{N} \operatorname{Id}_{N}-i \theta\right) \in S U(N),
$$


where $k=\overline{0, N-1}$. The function $\Phi_{0}([\theta], \lambda)$ is holomorphic, $\Phi_{N-1}([\theta], \lambda)$ is antiholomorphic and the remaining ones $k=\overline{1, N-2}$ are mixed. If $\lambda \rightarrow+\infty$, then $\Phi_{k}([\theta], \lambda)$ satisfies the on-shell condition (5.9).

For any real functions $f$ and $g$ of one variable, the equations of motion (9.2) admit the Lie symmetries given by the prolongations to $J^{2}$ of the vector fields

$$
X_{C}=\left[f(x) \partial_{x} \theta^{j}+g(y) \partial_{y} \theta^{j}\right] \frac{\partial}{\partial \theta^{j}},
$$

which are called conformal as they vanish on functions depending only on the independent variables $x, y$. The integrated form of the immersed surface in $\mathfrak{s u}(N)$ induced by the above Lie symmetry is given by the FG formula [30, p. 15]. Using (9.7) for $k=0$ and in view of Theorem 8.1, we obtain

$$
F^{F G}=\Phi^{-1}\left(f(x) \hat{U}_{x}+g(y) \hat{U}_{y}\right) \Phi \in \mathfrak{s u}(N) .
$$

Alternatively, the spectral $\mathfrak{s u}(N)$-valued differential form $\Upsilon:=\operatorname{Ad}_{\Phi}\left(-\left[\partial_{x} \theta, \theta\right] \mathrm{d} x+\right.$ $\left.\left[\partial_{y} \theta, \theta\right] \mathrm{d} y\right)$ satisfies $\mathbf{d}_{2 \omega} \Upsilon=0$ on-shell. In view of Lemma 6.2, it provides a deformation giving rise to an immersion surface of the form $\mathbf{d} F=-\left[\partial_{x} \theta, \theta\right] \mathrm{d} x+\left[\partial_{y} \theta, \theta\right] \mathrm{d} y$ and

$$
F(x, y)=\int_{\gamma}\left(-\left[\partial_{x} \theta, \theta\right] \mathrm{d} x+\left[\partial_{y} \theta, \theta\right] \mathrm{d} y\right) .
$$

This is indeed the expression in the set of coordinates $\{x, y\}$ on $\mathcal{R}$ of the Weirstrass immersion formula for $\mathbb{C} P^{N-1}$ models [39].

\subsection{Soliton surfaces associated with the $\mathbb{C} P^{2}$ sigma model}

Let us analyse the geometric properties of immersion surfaces for the particular $\mathbb{C} P^{N-1}$ sigma model with $N=3$. One may see that once immersion formulas have been determined using out theoretical techniques, their geometric analysis does not show any additional theoretical or practical complication (cf. [28,29,31]). In the present case, the obtained surface is one of the elements of the so-called Veronese sequence [6,15]. The projector $P$ for $k=0$ can be written through the holomorphic function $f_{0}:=\left(1, \sqrt{2} \xi, \xi^{2}\right)$. One obtains that

$$
P_{0}=\frac{f_{0} \otimes f_{0}^{\dagger}}{f_{0}^{\dagger} f_{0}}=\frac{1}{\left(1+|\xi|^{2}\right)^{2}}\left(\begin{array}{ccc}
1 & \sqrt{2} \bar{\xi} & \bar{\xi}^{2} \\
\sqrt{2} \xi & 2|\xi|^{2} & \sqrt{2}|\xi|^{2} \bar{\xi} \\
\xi^{2} & \sqrt{2}|\xi|^{2} \xi & |\xi|^{4}
\end{array}\right)
$$

Hence, $X_{0}=\mathrm{i}\left(\mathrm{Id}_{3} / 3-P_{0}\right)$ and therefore

$$
X_{0}=\frac{\mathrm{i}}{\left(1+|\xi|^{2}\right)^{2}}\left(\begin{array}{ccc}
\frac{1}{3}\left(|\xi|^{4}+2|\xi|^{2}-2\right) & -\sqrt{2} \bar{\xi} & -\bar{\xi}^{2} \\
-\sqrt{2} \xi & \frac{1}{3}\left(|\xi|^{4}-4|\xi|^{2}+1\right) & -\sqrt{2}|\xi|^{2} \bar{\xi} \\
-\xi^{2} & -\sqrt{2}|\xi|^{2} \xi & \frac{1}{3}\left(1-2|\xi|^{4}+2|\xi|^{2}\right)
\end{array}\right)
$$


This is an immersion surface which is an anti-Hermitian solution of the equation:

$$
X_{0}^{2}+\frac{\mathrm{i}}{3} X_{0}+\frac{2}{9} \mathrm{Id}_{3}=0 .
$$

By considering the basis $S_{i}$ for $\mathfrak{s u}(3)$ given by the orthonormalization of the basis in ( [32, p. 11]) relative to the Killing metric on $\mathfrak{s u}(3)$ of the form $\langle A, B\rangle:=-\operatorname{Tr}(A B) / 2$, we obtain that every $X_{0}=\sum_{\alpha=1}^{8} x_{\alpha} S_{\alpha}$ satisfying the matrix equation (9.10) is such that its coordinates obey the following independent equations:

$$
\begin{aligned}
& \sum_{i=1}^{8} x_{i}^{2}=\frac{1}{3}, \\
& \frac{4}{3}\left(x_{4}-\frac{1}{4 \sqrt{3}}\right)^{2}+\sum_{i=5}^{8} x_{i}^{2}=\frac{1}{4}, \\
& \sum_{i=1}^{3} x_{i}^{2}+\frac{1}{3} x_{4}^{4}+x_{6}^{2}+x_{8}^{2}+\frac{1}{3} x_{3}+\frac{1}{3 \sqrt{3}}\left(6 x_{3}+1\right) x_{4}=\frac{2}{9}, \\
& -3 x_{3}^{3}-6\left(\sqrt{3} x_{4}+1\right) x_{3}-9 x_{4}^{2}+2 \sqrt{3} x_{4}+1=0 .
\end{aligned}
$$

The first one follows from the trace of (9.10). The second and third ones come from analyzing the diagonal elements of (9.10). Other conditions, e.g.

$$
\frac{1}{3} x_{2}\left(2 \sqrt{3} x_{4}+1\right)+x_{6} x_{7}-x_{5} x_{8}=0, \frac{1}{3}\left(2 \sqrt{2} x_{4}+1\right) x_{1}-x_{5} x_{6}-x_{7} x_{8}=0
$$

arise from analyzing all entries of Eq. (9.10).

Previous conditions allow us to determine the shape of the immersed surface. Equation (9.11) represents a sphere in $\mathbb{R}^{8}$. The Veronese surface results of intersecting the sphere (9.11) by an ellipsoidal cylinder (9.12) (the generatrix lines parallel to the $\left(x_{1}, x_{2}, x_{3}\right)$ axes) and a degenerate hyperbola (9.13). Equation (9.12) shows that $-1 /(2 \sqrt{3}) \leq x_{4} \leq 1 / \sqrt{3}$, while (9.14) determines a discrete set of values of $x_{3}$ for those possible values of $x_{4}$. Once particular values of $x_{3}$ and $x_{4}$ are determined, Eqs. (9.11)-(9.14) show that the coordinates $x_{1}, x_{2}, x_{6}, x_{8}, x_{5}, x_{7}$ of our surface are contained in the two-dimensional spheres of the form

$$
x_{1}^{2}+x_{2}^{2}=F_{1}\left(x_{3}, x_{4}\right), \quad x_{6}^{2}+x_{8}^{2}=F_{2}\left(x_{3}, x_{4}\right), \quad x_{7}^{2}+x_{5}^{2}=F_{3}\left(x_{3}, x_{4}\right),
$$

for certain functions $F_{1}, F_{2}, F_{3}$. The final expression for the immersed surfaces can be obtained via the remaining conditions, e.g. (9.15), resulting from (9.10).

Let us analyse the immersed surface using its global and local invariants. The parametrization of the surface in terms of the complex variable $\xi=x+i y$ is conformal, namely the Lie derivative in terms of $\partial_{\bar{\xi}}$ of the fundamental form $I=4\left(1+x^{2}+y^{2}\right)^{-2}(\mathrm{~d} x \otimes \mathrm{d} x+\mathrm{d} y \otimes \mathrm{d} y)=2\left(1+|\xi|^{2}\right)^{-2}(\mathrm{~d} \xi \otimes \mathrm{d} \bar{\xi}+\mathrm{d} \bar{\xi} \otimes \mathrm{d} \xi)$ which is the metric of the sphere, vanishes. 
Some simple calculations give that the Gaussian curvature of our immersed surface is $\kappa=2$ while the Euler-Poincaré character is $\chi=2$. The mean curvature tensor of the surface, $\mathcal{H}$, is anti-Hermitian and orthogonal, relative to the metric on $\mathfrak{s u}(3)$, to $\partial X_{0}, \bar{\partial} X_{0}$, and $X_{0}$. The norm of $\mathcal{H}$ is 16 . Then, the Veronese surface has positive constant mean curvature. Meanwhile, the Willmore functional indicates that the winding number of a submanifold of the surface is at least equal to 2 . This happens for the projection by $\pi:\left(x_{1}, \ldots, x_{8}\right) \in \mathbb{R}^{8} \simeq \mathfrak{s u}(3) \mapsto\left(x_{4}, x_{6}, x_{8}\right) \in \mathbb{R}^{3}$ of the Veronese surface.

Let us analyse a second possible immersed surface induced by a different spectral solution. It is well known that $f_{1}:=\left(\partial P_{0}\right) f_{0}$ gives rise to a new solution $P_{1}$ of the $\mathbb{C} P^{2}$ model. In other words, the function $f_{1}=\left(-2 \bar{\xi}, \sqrt{2}\left(1-|\xi|^{2}\right), 2 \xi\right) /\left(1+|\xi|^{2}\right)$ gives rise to the particular solution

$$
P_{1}=\frac{f_{1} \otimes f_{1}^{\dagger}}{f_{1}^{\dagger} f_{1}}=\frac{1}{\left(1+|\xi|^{2}\right)^{2}}\left(\begin{array}{ccc}
2|\xi|^{2} & \sqrt{2}\left(|\xi|^{2}-1\right) \bar{\xi} & -2 \bar{\xi}^{2} \\
\sqrt{2}\left(|\xi|^{2}-1\right) \xi & \left(|\xi|^{2}-1\right)^{2} & -\sqrt{2}\left(|\xi|^{2}-1\right) \bar{\xi} \\
-2 \xi^{2} & -\sqrt{2}\left(|\xi|^{2}-1\right) \xi & 2|\xi|^{2}
\end{array}\right)
$$

This leads to the immersion formula

$$
X_{1}=-\mathrm{i}\left(P_{1}+2 P_{0}\right)+\mathrm{i} \operatorname{Id}_{3}=\frac{\mathrm{i}}{\left(1+|\xi|^{2}\right)^{2}}\left(\begin{array}{ccc}
|\xi|^{2}-1 & \sqrt{2} \bar{\xi} & 0 \\
-\sqrt{2} \xi & 0 & -\sqrt{2} \bar{\xi} \\
0 & -\sqrt{2} \xi & -\left(|\xi|^{2}-1\right)
\end{array}\right)
$$

which is an anti-Hermitian solution to $X_{1}^{3}+X_{1}=0$. In the already used Gell-Mann orthonormal basis $\left\{S_{\alpha}\right\}_{\alpha \in \overline{1,8}}$ of $\mathfrak{s u}(3)$, the coordinates of the above $X_{1}$ satisfy

$$
x_{3}=x_{4} / \sqrt{3}, \quad x_{6}=0, \quad x_{8}=0, \quad x_{1}^{3}+1=0 .
$$

Since $X_{1} \neq 0$, the set of equations describing a non-singular manifold reduce to

$$
\begin{aligned}
& x_{5}^{2}+x_{7}^{2}+\frac{2}{3} x_{4}^{2}=\frac{1}{2} \\
& \sum_{i=1}^{7} x_{i}^{2}-x_{6}^{2}=1, \quad x_{1}^{4}-\left(x_{2}^{2}+\frac{2}{3} x_{4}^{2}-\frac{1}{2}\right)^{2}=0 .
\end{aligned}
$$

Thus, the six previous independent conditions (9.16)-(9.18) on $\mathfrak{s u ( 3 ) , ~ w h i c h ~ i s ~ e i g h t - ~}$ dimensional, give rise to a surface. The condition (9.18) shows that the immersed surface is a closed fourth degree curve in the coordinates $x_{1}, x_{2}$ parametrized by $-\frac{\sqrt{3}}{2}<x_{4} \leq \frac{\sqrt{3}}{2}$, where the above bounds for $x_{4}$ are a consequence of (9.17).

The metric of the surface is that one of the sphere with Gaussian curvature $\kappa=2$. The mean curvature is $\langle\mathcal{H}, \mathcal{H}\rangle=4$. This implies a smaller Willmore functional $W=$ $2 \pi$ relative to the Veronese surface, but a topological charge $Q=0$. 
A last anti-holomorphic solution to the $\mathbb{C} P^{2}$ model can be found

$$
P_{2}:=i \operatorname{Id}_{3}-P_{0}-P_{1}=\frac{1}{1+|\xi|^{2}}\left(\begin{array}{ccc}
|\xi|^{4} & -\sqrt{2}|\xi|^{2} \bar{\xi} & \bar{\xi}^{2} \\
-\sqrt{2}|\xi|^{2} \xi & 2|\xi|^{2} & -\sqrt{2} \bar{\xi} \\
\xi^{2} & -\sqrt{2} \xi & 1
\end{array}\right)
$$

which leads to an immersed surface

$$
\begin{aligned}
X_{2}: & =-i\left(P_{2}+2\left(P_{0}+P_{1}\right)\right)+i \frac{5}{3} \operatorname{Id}_{3} \\
& =\frac{i}{\left(1+|\xi|^{2}\right)^{2}}\left(\begin{array}{ccc}
-\frac{1}{3}\left[1-2|\xi|^{4}+2|\xi|^{2}\right] & -\sqrt{2}|\xi|^{2} \bar{\xi} & \bar{\xi}^{2} \\
-\sqrt{2}|\xi|^{2} \xi & -\frac{1}{3}\left[1+|\xi|^{4}-4|\xi|^{2}\right] & -\sqrt{2} \bar{\xi} \\
\xi^{2} & -\sqrt{2} \xi & -\frac{1}{3}\left[-2+|\xi|^{4}+2|\xi|^{2}\right]
\end{array}\right) .
\end{aligned}
$$

As this surface is a linear combination of previous ones, its analysis will be skipped.

\section{Concluding remarks and outlook}

We have presented a cohomological approach to the study of immersed soliton surfaces for integral systems of PDEs through several generalizations of $\mathfrak{g}$-valued differential forms. This has revealed several properties of such surfaces while providing an extension of known methods to general soliton submanifolds for more general systems of integrable PDEs. Our work has also shed some light on the jet bundle approach to the study of immersed soliton surfaces. The $\mathbb{C} P^{N-1}$ sigma models have been studied through our techniques.

In the future, we first plan to investigate the stability of soliton surfaces and their observability in nature. This study would attempt to devise a perturbation theory and lead to the development of approximate solutions. Second, analytic expressions for surfaces may reveal qualitative features that might otherwise be difficult to detect numerically. In this respect, we aim to develop computer techniques for the visualization of soliton surfaces. It is also natural to ask how the integrable characteristics-Hamiltonian structures, conserved quantities, singularities structures and so on-are present on surfaces. Third, we are interested in problems whose soliton surfaces are well known experimentally but the associated physical system is not fully developed. Hence, we propose to use the variational problem of the geometric functional, i.e. the Willmore action functional, to compute the Euler-Lagrange equations determining the surface. This approach has been accomplished for biological membrane models obtained via the generalized Weierstrass representation [39], but not, to our knowledge, for immersion formulas for soliton surfaces using our approach. It would be also pertinent, in the context of $\mathbb{C} P^{N}$ sigma models, to further develop these models via the coherent states approach as performed in [31]. Finally, the cohomological approach may be further developed and the properties of the used cohomologies must be studied in detail, e.g. using a description of more general Poincaré Lemmas.

Acknowledgements A.M. Grundland was partially supported by the research Grant ANR-11LABX-0056LMHLabEX LMH (France) and from the NSERC (Canada). J. de Lucas and A.M. Grundland acknowledge 
partial support from Project MAESTRO DEC-2012/06/A/ST1/00256 of the National Science Center (Poland). This work was partially accomplished during the stay of A.M. Grundland and J. de Lucas at the École Normale Superieure de Cachan (CMLA). The authors would also like to thank CMLA for its hospitality and attention during their stay. Finally, we thank an anonymous referee for valuable comments to improve the paper.

Open Access This article is distributed under the terms of the Creative Commons Attribution 4.0 International License (http://creativecommons.org/licenses/by/4.0/), which permits unrestricted use, distribution, and reproduction in any medium, provided you give appropriate credit to the original author(s) and the source, provide a link to the Creative Commons license, and indicate if changes were made.

\section{References}

1. Ablowitz, M.J.: Nonlinear Phenomena. Springer, Berlin (1982)

2. Babelon, O., Bernard, D., Talon, M.: Introduction to Classical Integrable Systems (Cambridge Monographs on Mathematical Physics). Cambridge University Press, Cambridge (2006)

3. Biernacki, W., Cieslinski, J.L.: A compact form of the Darboux-Backlund transformation for some spectral problems in Clifford algebras. Phys. Lett. A 288, 167-172 (2001)

4. Bobenko, A.I.: Surfaces in terms of 2 by 2 matrices. Old and new integrable cases. In: Fordy, A.P., Wood, J.C. (eds.) Harmonic Maps and Integrable Systems. Aspects of Mathematics, vol. E 23. Vieweg+Teubner Verlag, Wiesbaden (1994)

5. Bobenko, A., Eitner, U.: Painlevé Equations in the Differential Geometry of Surfaces. Lecture Notes in Mathematics, vol. 1753. Springer, Berlin (2000)

6. Bolton, J., Jensen, G.R., Rigoli, M., Woodward, L.M.: On conformal minimal immersions of $S^{2}$ into $\mathbb{C} P^{n}$. Math. Ann. 279, 599-620 (1988)

7. Cartan, E.: Sur la structure des groupes infinis de transformation in Les systèmes différentiels en Involution. Gauthier-Villars, Paris (1953)

8. Chavolin, J., Joanny, J.F., Zinn-Justin, J.: Liquids at Interfaces. Elsevier, Amsterdam (1989)

9. Chen, F.F.: Introduction to Plasma Physics and Controlled Fusion. Plasma Physics, vol. 1. Plenum Press, New York (1983)

10. Cieśliński, J.: A generalized formula for integrable classes of surfaces in Lie algebras. J. Math. Phys. 38, 4255-4272 (1997)

11. Cieśliński, J.L.: Geometry of submanifolds derived from spin-valued spectral problem. J. Theor. Math. Phys. 137, 1396-1405 (2003)

12. Cieśliński, J.L.: Pseudospherical surfaces on time scales: a geometric deformation and the spectral approach. J. Phys. A 40, 12525-12538 (2007)

13. David, F., Ginsparg, P., Zinn-Justin, J.: Fluctuating Geometries in Statistical Mechanics and Field Theory. North-Holland, Amsterdam (1996)

14. Davydov, A.S.: Solitons in Molecular Systems. Kluwer, New York (1991)

15. Dillen, F.J.E., Verstraelen, L.C.A.: Handbook of Differential Geometry. North-Holland, Amsterdam (2000)

16. Din, A.M., Horváth, Z., Zakrzewski, W.J.: The Riemann-Hilbert problem and finite action $\mathbb{C} P^{N-1}$ solutions. Nucl. Phys. B 233, 269-288 (1984)

17. Din, A.M., Zakrzewski, W.: General class of solutions in the $\mathbb{C} P^{N-1}$ model. Nucl. Phys. B 174, 397-406 (1980)

18. Doliwa, A., Sym, A.: Constant mean curvature surfaces in $E^{3}$ as an example of soliton surfaces. In: Nonlinear Evolution Equations and Dynamical Systems. World Scientific, River Edge, pp. 111-117 (1992)

19. Eichenherr, H.: $S U(N)$ invariant nonlinear $\sigma$ models. Nucl. Phys. B 146, 215-223 (1978)

20. Fokas, A.S., Gel'fand, I.M.: Surfaces on Lie groups, on Lie algebras, and their integrability. Commun. Math. Phys. 177, 203-220 (1996)

21. Fokas, A.S., Gel'fand, I.M., Finkel, F., Liu, Q.M.: A formula for constructing infinitely many surfaces on Lie algebras and integrable equations. Sel. Math. 6, 347-375 (2000)

22. Giachetta, G., Mangiarotti, L., Sardanashvily, G.: Cohomology of the infinite-order jet space and the inverse problem. J. Math. Phys. 42, 4272-4282 (2001) 
23. Goldstein, P.P., Grundland, A.M.: Invariant recurrence relations for $C P^{N-1}$ models. J. Phys. A 43, 265206 (2010)

24. Golo, V.L., Perelomov, A.M.: Solution of the duality equations for the two-dimensional $S U(N)$ invariant chiral model. Phys. Lett. B 79, 112-113 (1978)

25. Gross, D.J., Piran, T., Weinberg, S.: Two-Dimensional Quantum Gravity and Random Surfaces. World Scientific, Singapore (1992)

26. Grundland, A.M.: Soliton surfaces in the generalized symmetry approach. Theor. Math. Phys. 188, 1322-1333 (2016)

27. Grundland, A.M., Levi, D., Martina, L.: On immersion formulas for soliton surfaces. Acta Polytech. 56, 180-192 (2016)

28. Grundland, A.M., Post, S.: Soliton surfaces associated with generalized symmetries of integrable equations. J. Phys. A 44, 165203 (2011)

29. Grundland, A.M., Post, S.: Surfaces immersed in Lie algebras associated with elliptic integrals. J. Phys. A 45, 015204 (2012)

30. Grundland, A.M., Post, S., Riglioni, D.: Soliton surfaces and generalized symmetries of integrable systems. J. Phys. A 47, 015201 (2014)

31. Grundland, A.M., Strasburger, A., Dziewa-Dawidczyk, D.: $\mathbb{C} P^{N}$ sigma models via the $S U(2)$ coherent states approach, Banach Center Publications, Polish Academy of Sciences, 50th seminar 'Sophus Lie' 113 (2018)

32. Grundland, A.M., Strasburger, A., Zakrzewski, W.J.: Surfaces immersed in $\mathfrak{s u}(N+1)$ Lie algebras obtained from the $\mathbb{C} P^{N}$ sigma models. J. Phys. A 39, 9187 (2006)

33. Grundland, A.M., Yurduşen, I.: On analytic descriptions of two-dimensional surfaces associated with the $\mathbb{C} P^{N-1}$ sigma model. J. Phys. A 42, 172001 (2009)

34. Guo, X.R.: Three new $(2+1)$-dimensional integrable systems and some related Darboux transformations. Commun. Theor. Phys. 65, 735-742 (2016)

35. Hélein, F.: Constant Mean Curvature Surfaces, Harmonic Maps and Integrable Systems. Lectures in Mathematics ETH Zürich. Birkhäuser, Basel (2001)

36. Hopf, H.: Über die Abbildungen der Dreidimensionalen Sphäre auf die Kugelfläche. Math. Ann. 104, 637-665 (1931)

37. Igonin, S., Krasilshchik, J.: On one-parametric families of Bcklund transformations. Adv. Stud. Pure Math. 37, 99-114 (2002)

38. Kobayashi, S., Nomizu, K.: Foundations of Differential Geometry, vol. I. (Wiley Classics Library). Wiley, New York (1996)

39. Konopelchenko, B.G., Landolfi, G.: Generalized Weierstrass representation for surfaces in multidimensional Riemann spaces. J. Geom. Phys. 29, 319-333 (1999)

40. Konopelchenko, B.G.: Induced surfaces and their integrable dynamics. Stud. Appl. Math. 96, 9-51 (1996)

41. Krasil'shchik, J., Verbovetsky, A.: Geometry of jet spaces and integrable systems. J. Geom. Phys. 61, 1633-1674 (2011)

42. Kruglikov, B., Lychagin, V.V.: Geometry of differential equations. In: Krupka, D., Saunders, D. (eds.) Handbook of Global Analysis, vol. 1214, pp. 725-771. Elsevier, Amsterdam (2008)

43. Landolfi, G.: New results on the Canham-Helfrich membrane model via the generalized Weierstrass representation. J. Phys. A 36, 11937-11954 (2003)

44. Manakov, S.V., Santini, P.M.: Inverse scattering problem for vectors fields and the Cauchy problem for the heavenly equations. Phys. Lett. A 359, 613-619 (2006)

45. Manton, N., Sutcliffe, P.: Topological Solitons (Cambridge Monographs on Mathematical Physics). Cambridge University Press, Cambridge (2004)

46. Marvan, M.: On the horizontal gauge cohomology and nonremovability of the spectral parameter. Acta Appl. Math. 72, 51-65 (2002)

47. May, J.P.: A Concise Course in Algebraic Topology. Chicago Lectures in Mathematics. University of Chicago Press, Chicago (1999)

48. Mikhailov, A.V.: Integrable Magnetic Models Soliton. Modern Problems in Condensed Matter, vol. 17, pp. 623-690. North-Holland, Amsterdam (1986)

49. Mikhailov, A.V., Shabat, A.B., Sokolov, V.V.: The symmetry approach to classification of integrable equations. What is integrability? In: Zakharov, V.E. (ed.) Nonlinear Dynamics, pp. 115-184. Springer, Berlin (1991) 
50. Nelson, D., Piran, T., Weinberg, S.: Statistical Mechanics of Membranes and Surfaces. World Scientific, Singapore (1992)

51. Olver, P.J.: Applications of Lie Groups to Differential Equations. Springer, New York (1993)

52. Ou-Yang, Z., Liu, J., Xie, Y.: Geometric Methods in Elastic Theory of Membranes in Liquid Crystal Phases. World Scientific, Singapore (1999)

53. Polchinski, J., Strominger, A.: Effective string theory. Phys. Rev. Lett. 67, 1681-1684 (1991)

54. Rogers, C., Schief, W.K.: Bäcklund and Darboux Transformations. Geometry and Modern Applications in Soliton Theory. Cambridge Texts in Applied Mathematics. Cambridge University Press, Cambridge (2000)

55. Safran, S.A.: Statistical Thermodynamics of Surfaces, Interfaces, and Membranes. Frontiers of Physics, vol. 90. Westview Press, Boulder (2003)

56. Sasaki, J.R.: General class of solutions of the complex Grassmannian and $\mathbb{C} P^{N-1}$ model. Phys. Lett. B 130, 69-72 (1983)

57. Sommerfeld, A.: Lectures on Theoretical Physics. Academic Press, New York (1952)

58. Sym, A.: Soliton surfaces. Lett. Nuovo Cimento 33, 394-400 (1982)

59. Sym, A.: Soliton surfaces and their applications (soliton geometry from spectral problems). In: Geometric Aspect of the Einstein Equation and Integrable Systems. Lectures Notes in Physics, vol. 239, pp. 154-231. Springer, Berlin (1995)

60. Tafel, J.: Surfaces in $\mathbb{R}^{3}$ with prescribed curvature. J. Geom. Phys. 17, 381-390 (1995)

61. Urbantke, H.K.: The Hopf fibration-seven times in physics. J. Geom. Phys. 46, 125-150 (2003)

62. Vinogradov, A.M.: Cohomological Analysis of Partial Differential Equations and Secondary Calculus. Translations of Mathematical Monographs, vol. 204. American Mathematical Society, Providence (2001)

63. Vinogradov, A.M., Krasil'shchik, I.S.: Symmetries and Conservation Laws for Differential Equations of Mathematical Physics. Translations of Mathematical Monographs, vol. 182. American Mathematical Society, Providence (1999)

64. Zakharov, V.E.: Dispersionless limit of integrable systems in $2+1$ dimensions. In: Ercolani, N.M., Gabitov, I.R., Levermore, C.D., Serre, D. (eds.) Singular Limits of Dispersive Waves. NATO Advanced Study Institute, Series B: Physics, vol. 320. Plenum, New York (1994)

65. Zakharov, V.E., Mikhailov, A.V.: Relativistically invariant two-dimensional models of field theory which are integrable by means of the inverse scattering problem method. Sov. Phys. JETP 74, 19531973 (1978)

66. Zakrzewski, W.J.: Low-Dimensional Sigma Models. Adam Hilger, Bristol (1989) 